\title{
Deficient Butyrate-Producing Capacity in the Gut Microbiome of Myalgic Encephalomyelitis/Chronic Fatigue Syndrome Patients is Associated with Fatigue Symptoms
}

Cheng Guo

Columbia University, Center for Infection and Immunity

Xiaoyu Che

Columbia University

Thomas Briese

Columbia University Mailman School of Public Health

Orchid Allicock

Columbia University

Rachel A. Yates

Columbia University

Aaron Cheng

Columbia University

Amit Ranjan

Columbia University

Dana March

Columbia University Mailman School of Public Health

Mady Hornig

Columbia University Mailman School of Public Health

Anthony L. Komaroff

Harvard Medical School

Susan Levine

Levine Clinic

Lucinda Bateman

Bateman Horne Center

Suzanne D. Vernon

Bateman Horne Center

Nancy G. Klimas

Nova Southeastern University - Fort Lauderdale/Davie Campus: Nova Southeastern University Jose G. Montoya

Palo Alto Medical Foundation 


\section{Daniel L. Peterson}

Sierra Internal Medicine at Incline Village

\section{W. lan Lipkin}

Columbia University Mailman School of Public Health

Brent L. Williams ( $\square$ bw2101@cumc.columbia.edu )

Columbia University Mailman School of Public Health https://orcid.org/0000-0003-2740-5051

\section{Research}

Keywords: Myalgic Encephalomyelitis/Chronic Fatigue Syndrome, microbiome, 86 butyrate, irritable bowel syndrome

Posted Date: October 29th, 2021

DOI: https://doi.org/10.21203/rs.3.rs-1017818/v1

License: (c) (i) This work is licensed under a Creative Commons Attribution 4.0 International License. Read Full License 
1 Deficient butyrate-producing capacity in the gut microbiome of Myalgic

2 Encephalomyelitis/Chronic Fatigue Syndrome patients is associated with fatigue

3 symptoms

4 Cheng Guo ${ }^{1}$, Xiaoyu Che ${ }^{1,2}$, Thomas Briese ${ }^{1,3}$, Orchid Allicock ${ }^{1}$, Rachel A. Yates ${ }^{1}$, Aaron

5 Cheng ${ }^{1}$, Amit Ranjan ${ }^{1}$, Dana March ${ }^{3}$, Mady Hornig ${ }^{3}$, Anthony L. Komaroff ${ }^{4}$, Susan

6 Levine $^{5}$, Lucinda Bateman ${ }^{6}$, Suzanne D. Vernon ${ }^{6}$, Nancy G. Klimas ${ }^{7,8}$, Jose G. Montoya ${ }^{9}$,

7 Daniel L. Peterson ${ }^{10}$, W. Ian Lipkin ${ }^{1,3,11}$, Brent L. Williams ${ }^{1,3,11^{*}}$

91 Center for Infection and Immunity, Mailman School of Public Health, Columbia

10 University, New York, NY, 10032, USA

$11{ }^{2}$ Department of Biostatistics, Mailman School of Public Health, Columbia University, New

12 York, NY, 10032, USA

$13{ }^{3}$ Department of Epidemiology, Mailman School of Public Health, Columbia University,

14 New York, NY, 10032, USA

$15{ }^{4}$ Division of General Medicine, Department of Medicine, Brigham and Women's

16 Hospital, Harvard Medical School, Boston, MA, 02115, USA

$17 \quad{ }^{5}$ Levine Clinic, New York, NY, 10021, USA

$18 \quad{ }^{6}$ Bateman Horne Center, Salt Lake City, UT, 84102, USA

$19{ }^{7}$ Institute for Neuro-Immune Medicine, College of Osteopathic Medicine, Nova

20 Southeastern University, Fort Lauderdale, FL, 33314, USA

$21{ }^{8}$ Miami VA Medical Center, Miami, FL, 33125, USA

$22{ }^{9}$ Palo Alto Medical Foundation, Jack S. Remington Laboratory for Specialty Diagnostics

23 of Toxoplasmosis, Palo Alto, CA, 94301, USA 
$25{ }^{10}$ Sierra Internal Medicine at Incline Village, Incline Village, NV, 89451, USA

2611 Department of Pathology and Cell Biology, College of Physicians and Surgeons,

27 Columbia University, New York, NY, 10032, USA

$29 \quad{ }^{*}$ Corresponding author: bw2101@cumc.columbia.edu

31 Email addresses for all authors:

32 Cheng Guo (cg2984@cumc.columbia.edu), Xiaoyu Che (xc2273@cumc.columbia.edu), 33 Thomas Briese (tb2047@cumc.columbia.edu), Orchid Allicock

34 (orchid.allicock@yale.edu), Rachel A. Yates (rachelay@gmail.com), Aaron Cheng 35 (aaron.cheng@caa.columbia.edu), Amit Ranjan (ar4111@cumc.columbia.edu), Dana 36 March (dm2025@cumc.columbia.edu), Mady Hornig (mh2092@cumc.columbia.edu), 37 Anthony L. Komaroff (Anthony_komaroff@hms.harvard.edu), Susan Levine 38 (cfssuelev@earthlink.net), Lucinda Bateman (LBateman@batemanhornecenter.org), 39 Suzanne D. Vernon (sdvernon@batemanhornecenter.org), Nancy G. Klimas 40 (nklimas@nova.edu), Jose G. Montoya (montoyj@sutterhealth.org), Daniel L. Peterson 41 (DPeterson@sierrainternalmed.com), W. Ian Lipkin (wil2001@cumc.columbia.edu), 42 Brent L. Williams (bw2101@cumc.columbia.edu) 


\section{Abstract}

48 Background: Myalgic Encephalomyelitis/Chronic Fatigue Syndrome (ME/CFS) is a

49 complex, debilitating disease of unknown cause for which there is no specific therapy.

50 Patients suffering from ME/CFS commonly experience persistent fatigue, post-exertional

51 malaise, cognitive dysfunction, sleep disturbances, orthostatic intolerance, fever and

52 irritable bowel syndrome (IBS). Recent evidence implicates gut microbiome dysbiosis in

53 ME/CFS. However, most prior studies are limited by small sample size, differences in

54 clinical criteria used to define cases, limited geographic sampling, reliance on bacterial

55 culture or $16 \mathrm{~S}$ rRNA gene sequencing, or insufficient consideration of confounding factors

56 that may influence microbiome composition. In the present study, we evaluated the fecal

57 microbiome in the largest prospective, case-control study to date ( $n=106$ cases, $n=91$

58 healthy controls), involving subjects from geographically diverse communities across the

59 United States.

60 Results: Using shotgun metagenomics and qPCR and rigorous statistical analyses that

61 controlled for important covariates, we identified decreased relative abundance and

62 quantity of Faecalibacterium, Roseburia, and Eubacterium species and increased

63 bacterial load in feces of subjects with ME/CFS. These bacterial taxa play an important

64 role in the production of butyrate, a multifunctional bacterial metabolite that promotes

65 human health by regulating energy metabolism, inflammation, and intestinal barrier

66 function. Functional metagenomic and qPCR analyses were consistent with a deficient

67 microbial capacity to produce butyrate along the acetyl-CoA pathway in ME/CFS.

68 Metabolomic analyses of short-chain fatty acids (SCFAs) confirmed that fecal butyrate

69 concentration was significantly reduced in ME/CFS. Further, we found that the degree of 
70 deficiency in butyrate-producing bacteria correlated with fatigue symptom severity among

71 ME/CFS subjects. Finally, we provide evidence that IBS comorbidity is an important

72 covariate to consider in studies investigating the microbiome of ME/CFS subjects, as

73 differences in microbiota alpha diversity, some bacterial taxa, and propionate were

74 uniquely associated with self-reported IBS diagnosis.

75 Conclusions: Our findings indicate that there is a core deficit in the butyrate-producing 76 capacity of the gut microbiome in ME/CFS subjects compared to healthy controls. The

77 relationships we observed among symptom severity and these gut microbiome 78 disturbances may be suggestive of a pathomechanistic linkage, however, additional

79 research is warranted to establish any causal relationship. These findings provide support

80 for clinical trials that explore the utility of dietary, probiotic and prebiotic interventions to 81 boost colonic butyrate production in ME/CFS.

82

85 Keywords: Myalgic Encephalomyelitis/Chronic Fatigue Syndrome, microbiome, 86 butyrate, irritable bowel syndrome 


\section{Background}

94 Myalgic Encephalomyelitis/Chronic Fatigue Syndrome (ME/CFS) is an unexplained

95 debilitating and chronic disease characterized by a spectrum of symptoms including 96 fatigue, post-exertional malaise, impaired memory, pain, gastrointestinal dysfunction, 97 immune abnormalities, and sleep disturbances [1-3]. The global prevalence of ME/CFS 98 ranges between 0.4 and $2.5 \%$. The illness predominantly begins in adults 20 to 40 years 99 of age, and is more common in females than males with a ratio averaging about 3:1, 100 ranging as high as 6:1[4-7]. In the US alone this syndrome afflicts 2.5 million individuals 101 [3], with an estimated economic burden of approximately \$18 - \$24 billion USD [8]. A diagnosis of ME/CFS is based on clinical criteria and symptoms utilizing different

103 but overlapping case definitions including the 1994 U.S. Centers for Disease Control and 104 Prevention (CDC), Canadian Consensus, International Consensus, and the Oxford and 105 Institute of Medicine Criteria [1-3, 9, 10]. The cause or causes of ME/CFS are unknown. 106 However, many underlying biological abnormalities have been identified in people with 107 ME/CFS, including defective energy metabolism and a hypometabolic state; redox 108 imbalance; dysregulated immune responses; multiple abnormalities of the central and 109 autonomic nervous system; and multiple autoantibodies, many against targets in the 110 central and autonomic nervous system, as summarized in two recent reviews [11, 12].

111 Many, but not all, people with ME/CFS report that their symptoms began following 112 an acute prodromal viral-like syndrome [10]. Post-infection fatigue states are known to 113 develop following viral, bacterial and protozoal infections [11]. Clusters of ME/CFS have 114 also followed outbreaks of infectious disease $[13,14]$. Interestingly, recent viral outbreaks 115 including SARS-CoV, Ebola, MERS-CoV and SARS-CoV-2 have been associated with 
116 long-term sequelae that include persistent fatigue and cognitive symptoms consistent with

117 those reported in ME/CFS [15-19].

118 The gut microbiome can influence human health and affect physiological systems

119 through its influence on pathogen resistance, maintenance of the gut barrier, metabolism,

120 immunity, and neural signaling. The gut microbiome directly affects immunity via the

121 production of various immunomodulatory metabolites such as short-chain fatty acids

122 (SCFAs), which support immunological tolerance and maintain inflammatory equilibrium

123 [20-23]. Several studies, including our own, have identified differences in the gut

124 microbiome (dysbiosis) between ME/CFS patients and healthy individuals [24-30].

125 However, most of these studies have limitations due to small sample size, insufficient

126 assessment of potentially confounding variables, and biases associated with $16 \mathrm{~S}$

127 sequencing for bacterial classification.

128 In this study, we employed fecal shotgun metagenomics and metabolomics to 129 assess dysbiosis in the largest prospective case-control study to date, consisting of 106

130 ME/CFS subjects and 91 matched healthy controls. Our findings provide new insights into 131 disturbances in the microbiota, functional metagenomic pathways, and SCFAs and their 132 relationship with fatigue symptoms in ME/CFS.

134 Results

135 Study Population

136 The study included stool samples from 106 ME/CFS cases who met both the 1994 CDC

137 and the 2003 Canadian consensus criteria for ME/CFS, and 91 healthy control individuals

138 recruited from 5 sites in the United States. The characteristics of the ME/CFS patients 
139 and healthy participants of this study are outlined in Table 1. The majority of the ME/CFS 140 cases were categorized as long duration, with 92.5\% presenting with MECFS for longer

141 than 3 years. Self-reported irritable bowel syndrome diagnosis (sr-IBS) was more frequent 142 in ME/CFS cases (33.0\%) compared to healthy controls (3.3\%) (Fisher's Exact test, $\mathrm{p}<$ 143 0.001), consistent with prior reports of higher IBS comorbidity in ME/CFS [31-33].

144 The site of collection, age, sex, BMI, and demographic index were matched in 145 healthy controls. More ME/CFS females enrolled in the study $(70.8 \%$ of all ME/CFS 146 cases), consistent with a higher prevalence of ME/CFS in females in the general 147 population. Healthy controls consisted of $75.8 \%$ females. Mean age was similar between $148 \mathrm{ME} / \mathrm{CFS}$ and healthy controls, 47.8 vs 47.0 years, respectively. Mean BMI was also 149 similar between cases and controls, and $50 \%$ of the cases and $52.7 \%$ of the controls had 150 a high BMI (over $25 \mathrm{~kg} / \mathrm{m}^{2}$ ).

151 No cases or controls took an antibiotic in the six weeks prior to stool sample 152 collection (see participant exclusion criteria), and antibiotic use in the 6-12 week period 153 prior to sample collection was similar between cases and controls. As prebiotic and 154 probiotic supplement use can influence the microbiome, we compared usage between 155 cases and controls. More ME/CFS subjects reported frequent prebiotic (Fisher's Exact 156 test, $p=0.02$ ) and probiotic (Fisher's Exact test, $\mathrm{p}<0.001$ ) supplement usage compared 157 to healthy controls.

\section{Characterization of the gut microbiome}

160 Shotgun metagenomic sequencing of stool samples from all $106 \mathrm{ME} / \mathrm{CFS}$ and 91 healthy 161 controls was undertaken to assess bacterial composition, alpha and beta diversity, 
162 differential abundance of bacterial taxa, and predicted functional metabolic pathways. On

163 average, >27 million (range: 12-68 million) raw sequences were obtained per sample.

164 After quality filtering and host subtraction, bacterial reads were classified using Kraken2

165 and relative abundances of bacteria were estimated using Bracken. At the genus-level,

166 across the dataset, Bacteroides was the most abundant genus in both ME/CFS cases

167 and healthy controls, consistent with prior reports of Bacteroides dominance in individuals

168 in the United States (Figure 1A) [34]. Other high abundance genera observed in ME/CFS

169 and healthy controls included Alistipes, Parabacteroides, Roseburia, Prevotella, and

170 Faecalibacterium.

171 Bacterial alpha diversity metrics (Pielou's evenness, Shannon index, and observed

172 species) were compared in univariate analyses to assess differences in alpha diversity

173 between ME/CFS cases and healthy controls. The microbiota of ME/CFS patients had

174 lower evenness (Mann-Whitney U test, $p=0.024$ ) and Shannon diversity (Mann-Whitney

$175 U$ test, $p=0.023$ ) (Figure 1B and Supplementary Figure 1A) but similar observed

176 species (Supplementary Figure 1B) compared to healthy controls.

177 IBS has also been associated with changes in the gut microbiota, including alpha

178 diversity [35-38]. Given that the frequency of sr-IBS diagnosis was higher in ME/CFS in

179 this study, and that our prior research indicated that comorbid sr-IBS may be associated

180 with alterations in microbiota in ME/CFS [25], we performed stratified analyses to assess

181 differences in alpha diversity among healthy controls without sr-IBS ( $n=88)$, ME/CFS

182 without sr-IBS $(n=71)$ and ME/CFS with sr-IBS $(n=35)$. As only three healthy controls had

183 sr-IBS diagnosis in our cohort, no stratified comparisons with this group were undertaken.

184 In stratified analyses, evenness and Shannon diversities were only lower in ME/CFS with 
185 sr-IBS compared with healthy controls without sr-IBS (Mann-Whitney $U$ test, $p^{\text {adj }}=0.038$

186 and $\mathrm{p}^{\mathrm{adj}}=0.047$, respectively) (Figure 1C and Supplementary Figure 1C). No

187 differences were found between stratified groups for observed species (Supplementary

188 Figure 1D). These results indicate that differences in alpha diversity between ME/CFS

189 and controls may be dependent on comorbid sr-IBS, rather than ME/CFS.

190 To further evaluate the relationship between alpha diversity and ME/CFS, we 191 employed linear regression (Shannon and evenness) and negative binomial regression

192 (observed species) with alpha diversity metrics as outcome variables and ME/CFS and 193 sr-IBS as predictors, adjusting for covariates of site of sampling, sex, BMI, race/ethnicity,

194 age, antibiotic usage 6-12 weeks prior to sample collection, probiotic supplement use, 195 and prebiotic supplement use (Table 2). The interaction term between ME/CFS and sr196 IBS was explored but was not included in the final model as it was not significant in any 197 of the regression models for alpha diversity. ME/CFS was not a significant predictor of 198 evenness, Shannon diversity, or observed species metrics. However, sr-IBS status was 199 negatively associated with evenness ( $\left.\beta_{\text {Est }}=-0.03,95 \% \mathrm{Cl}:-0.06-0.00, p=0.032\right)$ and 200 showed a trending negative association with Shannon diversity $\left(\left(\beta_{\mathrm{Est}}=-0.03,95 \% \mathrm{Cl}\right.\right.$ : $2010.06-0.00, p=0.054)$. Of other covariates, only subject age showed a significant positive 202 association with all three alpha diversity metrics. These results suggest that differences 203 in alpha diversity are associated with sr-IBS rather than ME/CFS and highlight the 204 importance of assessing IBS as a potential confounder of microbiota differences in 205 ME/CFS.

206 Differences in microbiota beta diversity based on the Bray-Curtis dissimilarity 207 between ME/CFS and healthy controls were assessed by PERMANOVA (unadjusted) 
208 and PERMANOVA-FL (adjusted for sr-IBS status, site of sampling, sex, BMI,

209 race/ethnicity, age, antibiotic usage 6-12 weeks prior to sample collection, probiotic

210 supplement use, and prebiotic supplement use). Although PCoA plots did not show clear

211 separation of cases and controls, ME/CFS subjects differed from healthy controls based

212 on Bray-Curtis dissimilarity even after adjusting for covariates (PERMANOVA, $p=0.002$;

213 PERMANOVA-FL, $p=0.025$ ) (Figure 1D). We performed stratified analyses based on

214 sr-IBS diagnosis to assess the possibility that differences in microbiota beta diversity were

215 driven by sr-IBS diagnosis (Figure 1E). Unlike alpha diversity, beta diversity differed

216 between healthy controls without sr-IBS and both ME/CFS without sr-IBS (PERMANOVA,

$\left.217 \mathrm{p}^{\text {adj }}=0.009\right)$ and ME/CFS with sr-IBS (PERMANOVA, $\left.\mathrm{p}^{\text {adj }}=0.015\right)$. Beta diversity did not

218 differ between ME/CFS without and with sr-IBS. Thus, beta diversity differences between

219 ME/CFS and healthy controls are independent of sr-IBS diagnosis.

220 Our results suggest that whereas some differences in microbiota between ME/CFS

221 and healthy controls may be confounded by sr-IBS diagnosis, other differences may be

222 independent of sr-IBS and specific to ME/CFS. Thus, we employed both unstratified and

223 stratified analyses and include sr-IBS diagnosis as a covariate in all regression models.

225 Differential abundance of bacterial taxa

226 We employed Generalized Additive Models for Location, Scale and Shape with a zero-

227 inflated beta distribution (GAMLSS-BEZI) to evaluate bacterial taxa that differ in relative

228 abundance in ME/CFS [39]. To identify differentially abundant species that are unique to

229 ME/CFS as well as those that are unique to ME/CFS with sr-IBS, we compared four

230 models. Model 1 represented the unstratified comparison of all ME/CFS vs. healthy 
231 controls. Model 2 represented the stratified comparison of ME/CFS without sr-IBS vs.

232 healthy controls without sr-IBS. Model 3 represented the stratified comparison of ME/CFS

233 with sr-IBS vs. healthy controls without sr-IBS. Model 4 represented the stratified

234 comparison of ME/CFS with sr-IBS vs. ME/CFS without sr-IBS (note; Model 4 did not

235 produce any significant taxa differences and is therefore not shown). For each of Models

236 1-3 ME/CFS status was the variable for comparison, while sr-IBS status was the variable

237 for comparison in Model 4, and each model is adjusted for covariates.

238 At the species-level, Model 1 identified four species after adjusting for multiple

239 comparisons that differentiated ME/CFS from healthy controls. Model 2 identified two

240 species that differentiated ME/CFS without sr-IBS from healthy controls without sr-IBS.

241 Model 3 identified fifteen species that differentiated ME/CFS with sr-IBS from healthy

242 controls without sr-IBS (Figure 2A and Supplementary Table 1A). The relative

243 abundance of two species, Eubacterium rectale and Faecalibacterium prausnitzii were

244 lower in the ME/CFS group in all three models. These results suggest that deficiency of

245 these species in ME/CFS are independent of sr-IBS and other covariates. The most

246 differentially abundant species were found in Model 3, where fifteen species were

247 associated with ME/CFS with sr-IBS compared to healthy controls without sr-IBS. The

248 majority of identified species (11/15) in Model 3 showed no overlap with other models

249 suggesting that sr-IBS has an independent association with changes in the microbiota.

250 The species associated with ME/CFS with sr-IBS included decreased relative abundance

251 of Alistipes putredinis, Dorea longicatena, Odoribacter splanchnicus, and

252 Lachnospiraceae bacterium GAM79, among others, and increased relative abundance of

253 Clostridium bolteae and Flavonifractor plautii. It was also apparent from our analysis of 
254 Model 1 that several bacterial species from the same genera such as Eubacterium and

255 Roseburia were significantly associated with ME/CFS before FDR adjustment

256 (Supplementary Table 1A). Accordingly, we performed the same GAMLSS-BEZI

257 analyses at the genus level.

258 At the genus-level six bacterial genera were significantly associated with ME/CFS

259 from all three models after FDR adjustment (Figure 2B and Supplementary Table 1B).

260 The majority of these genera had lower relative abundance in the ME/CFS group

261 compared to healthy controls, including Eubacterium, Faecalibacterium, Dorea,

262 Roseburia and Gemmiger. Only Lachnoclostridium was increased in relative abundance

263 in the ME/CFS groups.

264 At both the species- and genus-level the primary bacteria identified in all three

265 models, and thus associated with ME/CFS independent of sr-IBS, contained the most

266 abundant and common butyrate-producing bacteria (BPB) in the human gut, including the

267 species E. rectale, F. prausnitzii and the genera Eubacterium, Faecalibacterium and 268 Roseburia.

270 Quantitation of BPB in fecal samples

271 While shotgun metagenomics can provide compositional information on individual fecal

272 bacteria, relative abundance of each taxon is dependent on the relative abundance of all

273 bacterial taxa in the microbiota and is not strictly quantitative. In order to assess

274 quantitative differences in fecal BPB, we carried out QPCR analysis using assays

275 targeting the 16S rRNA genes of Roseburia-Eubacterium genera and the species $F$.

276 prausnitzii to evaluate the quantity of these bacterial taxa per gram of feces. Compared 
277 to healthy controls, ME/CFS subjects had significantly fewer 16S rRNA gene copies of 278 Roseburia-Eubacterium genera (Mann-Whitney $U$ test, $p=0.0008$ ) (Figure 2C) and the 279 species F. prausnitzii (Mann-Whitney $U$ test, $p=0.004$ ) (Figure 2D) per gram of feces. In 280 stratified analyses, Roseburia-Eubacterium 16S copies/gram of feces were lower in both 281 ME/CFS subjects without sr-IBS (Mann-Whitney $U$ test, $p^{\text {adj }}=0.029$ ) and ME/CFS 282 subjects with sr-IBS (Mann-Whitney $U$ test, $p^{\text {adj }}=0.011$ ) compared to healthy controls 283 without sr-IBS (Figure 2E). Similarly, lower quantities of $F$. prausnitzii 16S/gram of feces 284 were found when comparing ME/CFS without sr-IBS (Mann-Whitney $U$ test, $\left.p^{\text {adj }}=0.079\right)$ 285 and ME/CFS with sr-IBS (Mann-Whitney $U$ test, $p^{\text {adj }}=0.018$ ) compared with healthy 286 controls without sr-IBS, though only a trend was observed in the former after adjusting for 287 multiple comparisons (Figure 2F).

As total bacterial load in stool has never been evaluated in ME/CFS, we carried 289 out qPCR using a broad range 16S rRNA gene-targeting assay. In contrast to the 290 quantitatively measured deficiencies in BPB in ME/CFS, ME/CFS subjects had higher 291 quantities of total $16 \mathrm{~S}$ copies/gram of feces compared to healthy controls (Mann-Whitney $292 U$ test, $p<0.0001$ ) (Figure 2G). Stratified analyses revealed that ME/CFS subjects 293 without sr-IBS had higher quantities of total bacterial 16S/gram of feces compared with 294 healthy controls without sr-IBS (Mann-Whitney U test, $p^{\text {adj }}<0.0001$ ), while other group 295 comparisons did not differ after adjusting for multiple comparisons (Figure 2H).

296 Since we measured both the quantity of BPB 16 S and total bacterial 16 S, we also 297 calculated the relative abundance of Roseburia-Eubacterium and F. prausnitzii (i.e., 298 Roseburia-Eubacterium 16S/total bacterial 16S). For both Roseburia-Eubacterium and F. 299 prausnitzii, ME/CFS subjects had lower calculated relative abundance compared with 
300 healthy controls (Mann-Whitney $U$ test, $p=0.0007$ and $p=0.009$, respectively)

301 (Supplementary Figure 2A, B). In stratified analyses, Roseburia-Eubacterium

302 calculated relative abundance was lower in ME/CFS subjects without sr-IBS compared 303 with healthy controls without sr-IBS (Mann-Whitney $U$ test, $p^{\text {adj }}=0.004$ ), while other 304 comparisons did not reach significance after adjusting for multiple comparisons

305 (Supplementary Figure 2C). Similar results were obtained for $F$. prausnitzii calculated 306 relative abundance, although only a trend toward lower $F$. prausnitzii was observed 307 comparing ME/CFS subjects without sr-IBS with healthy controls without sr-IBS (Mann308 Whitney $U$ test, $\left.p^{\text {adj }}=0.054\right)($ Supplementary Figure 2D).

309 To further assess the relationships between quantitative measures of BPB and 310 total bacteria (outcome variables) in feces with ME/CFS status (main predictor), 311 generalized linear regression models with a Gamma distribution with log link were fit 312 adjusting for covariates (Table 3). The estimated coefficients are interpreted as the log of 313 fold-change (FC). ME/CFS status was significantly associated with lower quantities of 314 Roseburia-Eubacterium 16S/gram of feces ( $\mathrm{FC}=0.56,95 \% \mathrm{Cl}: 0.33-0.93, \mathrm{p}=0.026)$ and 315 F. prausnitzii 16S/gram of feces ( $\mathrm{FC}=0.61,95 \% \mathrm{Cl}: 0.38-0.98, \mathrm{p}=0.043)$ and higher 316 quantities of total bacterial $16 \mathrm{~S} / \mathrm{gram}$ of feces ( $\mathrm{FC}=2.08,95 \% \mathrm{Cl}: 1.52-2.83)$. No other 317 covariates were associated with Roseburia-Eubacterium quantity in the regression 318 model, while BMI and antibiotic use were associated with F. prausnitzii quantity and BMI, 319 antibiotic use and site of patient recruitment (Florida) were associated with total bacterial 320 16S. Similar results were obtained in regression models assessing the measured relative 321 abundance of Roseburia-Eubacterium ( $F C=0.62,95 \% \mathrm{Cl}: 0.42-0.92, p=0.019)$ and $F$. 322 prausnitzii $(\mathrm{FC}=0.60,95 \% \mathrm{Cl}: 0.42-0.85, \mathrm{p}=0.004)$ (Supplementary Table 2). 
The findings indicate that, despite having higher quantities of total bacterial $16 \mathrm{~S}$ in

324 feces, ME/CFS patients had significantly lower quantities of important BPB such as the 325 genera Roseburia and Eubacterium and the species F. prausnitzii.

327 Fecal bacterial functional metagenomic pathways differ between ME/CFS and 328 healthy controls

329 Functional metagenomic analysis was carried out using GOmixer, a human gut 330 microbiome-specific metabolic pathway analysis tool [40]. Comparison between ME/CFS 331 subjects and healthy controls revealed nine global metabolic processes that differed 332 between the gut microbiome of ME/CFS subjects and controls (Figure $3 \mathrm{~A}$ and 333 Supplementary Table 3A). Two processes, butyrate and sulfate metabolism, were 334 deficient in ME/CFS. Seven processes, $\mathrm{CO}_{2}$ metabolism, monosaccharide degradation, 335 acetogenesis, ethanol production, cross-feeding intermediate metabolism, sugar acid 336 degradation and mucus degradation, were enriched in ME/CFS. At the level of gut 337 metabolic modules, whereas six modules were deficient, fifteen modules were enriched 338 (Supplementary Figure 3 and Supplementary Table 3B). The specific module for 339 butyrate production via transferase (MF0116) was deficient in ME/CFS, consistent with 340 deficiencies in BPB we observed based on differential abundance and qPCR analyses.

341 Other modules that were deficient in the ME/CFS microbiome included superoxide 342 reductase (MF0132) and sorbitol degradation (MF0073). Modules that were enriched in 343 ME/CFS included lactate production (MF0119), pyruvate dehydrogenase complex 344 (MF0083), ribose degradation (MF0060), and menaquinone production (MF0133).

345 Analysis of GOmixer gut-brain modules also revealed deficient metagenomic content for 
346 Butyrate synthesis II (MGB053), along with CIpB (ATP-dependent chaperone protein)

347 (MGB029), and S-Adenosylmethionine (SAM) synthesis (MGB036) (Supplementary

348 Table 3C). Enriched gut-brain modules in ME/CFS included Menaquinone synthesis

349 (vitamin K2) I (MGB040), GABA synthesis III (MGB022), and Isovaleric acid synthesis I

350 (KADH pathway) (MGB034).

351 As differential abundance analysis of taxa, qPCR, and functional metagenomic 352 analysis implicated deficiencies in BPB and functional capacity of the gut microbiome to 353 produce butyrate in ME/CFS subjects relative to healthy controls, we investigated the 354 metagenomic gene content for genes along the four pathways of gut bacterial butyrate 355 production: the acetyl-CoA pathway, the glutarate pathway, the lysine pathway, and the 356 4-aminobutyrate pathway (Figure 3B). We aligned bacterial reads to a curated database 357 of genes involved in butyrate production along the four pathways [41]. Comparing the 358 metagenomic content (Counts per Million, CPM) of each gene along the acetyl-CoA 359 pathway showed deficient gene content for nearly every gene along this pathway in 360 ME/CFS relative to healthy controls (Mann-Whitney $U$ test, $p<0.05$ for all genes except 361 thl and buk) (Figure 3C). Quantitatively, the majority of bacteria in the intestine encode 362 and utilize but (butyryl-CoA:acetate CoA transferase) rather than buk (butyrate kinase) as 363 the terminal gene in the acetyl-CoA pathway to produce butyrate. In fact, most bacteria 364 of the Eubacterium, Roseburia, and Faecalibacterium genera (those we found to be 365 deficient in ME/CFS) only encode the but gene [41]. While nearly all genes of the acetyl366 CoA pathway differed between ME/CFS and healthy controls, only terminal genes of the 367 glutarate pathway $(\operatorname{gcd} A$ and $\operatorname{gcd} B)$, and both early and terminal genes of the lysine 368 pathway (kamA, kamD, kamE, atoA, atoD) were deficient in ME/CFS compared to healthy 
369 controls, and no genes differed in the 4-aminobutyrate pathway in univariate analyses

370 (Supplementary Figure 4A-C). Even in stratified analyses by sr-IBS status, most genes

371 along the acetyl-CoA pathway remained significantly depleted in the microbiome of

372 ME/CFS subjects without sr-IBS compared with healthy controls without sr-IBS (Mann-

373 Whitney $\mathrm{U}$ test, $\mathrm{p}^{\mathrm{adj}}=0.071,0.038,0.035,0.049,0.020$ for $b h b d$, cro, etfA, etfB, and but,

374 respectively) (Figure 3D). In contrast, no genes along the glutarate, lysine, or 4-

375 aminobutyrate pathways differed significantly among groups in stratified analyses

376 (Supplementary Figure 4D-F).

377 We further evaluated metagenomic gene content for genes along butyrate

378 pathways using generalized linear regression with a Gamma distribution with log link and

379 adjusting for covariates. Even after adjusting for covariates, ME/CFS was a significant

380 predictor of most genes along the acetyl-CoA pathway (all except thl and buk) (Table 4).

381 Specifically, quantities of bhbd, cro, bcd, etfA, etfB and but were 0.81 -fold ( $95 \% \mathrm{Cl}$ : 0.68 -

$3820.96, p=0.014), 0.80$-fold $(95 \% \mathrm{Cl}: 0.68-0.95, p=0.008), 0.85$-fold $(95 \% \mathrm{Cl}: 0.73-1.00$,

$383 p=0.043), 0.84$-fold $(95 \% \mathrm{Cl}: 0.74-0.95, p=0.005), 0.85$-fold $(95 \% \mathrm{Cl}: 0.75-0.96, p=$

$3840.007)$ and 0.75 -fold (95\% Cl: $0.62-0.91, p=0.003)$ lower in ME/CFS than in controls,

385 respectively. In addition to ME/CFS status, BMI was associated with only but gene

386 content. In contrast, only a few genes in the glutarate pathway were associated with

387 ME/CFS status ( $g c t A, h g C o A d C$ and $g c d A$ ) in regression analyses (Supplementary

388 Table 4A). Only the terminal genes, ato $A$ and atoD, were associated with ME/CFS status

389 in the lysine pathway. Interestingly, most genes along the lysine pathway were

390 independently associated with race/ethnicity (Supplementary Table 4B). None of the 
391 genes in the 4-aminobutyrate pathway were significantly associated with ME/CFS status

392 (Supplementary Table 4C).

\section{Quantitation of the bacterial but gene in feces}

395 But, as the dominant terminal gene in the bacterial acetyl-CoA pathway for butyrate 396 production, can serve as an indicator gene for the overall butyrate-producing capacity in 397 the human gut [42]. We quantitated the but gene in fecal samples from ME/CFS and 398 control subjects by qPCR. The overall but copies/gram feces were lower in ME/CFS 399 compared to control subjects (Mann-Whitney $U$ test, $p=0.0003$ ) (Figure 3E). Further, 400 stratified analyses by sr-IBS status revealed lower but copies/gram feces in ME/CFS 401 without sr-IBS (Mann-Whitney $U$ test, $\left.p^{\text {adj }}=0.014\right)$ and ME/CFS with sr-IBS (Mann402 Whitney $U$ test, $p^{\text {adj }}=0.008$ ) compared to healthy controls without sr-IBS (Figure 3F). No 403 differences were found comparing ME/CFS subjects with and without sr-IBS. These 404 results suggest that deficient but gene quantity in the feces of ME/CFS patients is 405 independent of sr-IBS status.

406 We also assessed the relative abundance of but gene copies to total bacterial 16S 407 in feces based on qPCR. As with the total quantity of but gene/gram of feces, the but 408 gene calculated relative abundance was lower in ME/CFS subjects compared to healthy 409 controls in univariate analyses (Mann-Whitney $U$ test, $p=0.0003$ ) (Supplementary 410 Figure 5A). In stratified analyses by sr-IBS status, but gene relative abundance was lower

411 in ME/CFS subjects without sr-IBS compared with healthy controls without sr-IBS (Mann412 Whitney $U$ test, $p^{\text {adj }}=0.002$ ). A trend toward lower but gene relative abundance was 
413 observed in ME/CFS subjects with sr-IBS compared with healthy controls without sr-IBS

414 (Mann-Whitney $U$ test, $\left.\mathrm{p}^{\text {adj }}=0.096\right)($ Supplementary Figure 5B).

415 To further assess the relationships between quantitative measures of the but gene 416 in feces (outcome variable) with ME/CFS status (main predictor) generalized linear

417 regression models with a Gamma distribution with log link were assessed, adjusting for 418 covariates (Table 3). Even after adjusting for covariates in the model, ME/CFS was 419 associated with but gene quantities ( $F C=0.51,95 \% \mathrm{Cl}: 0.28-0.93, p=0.028)$. Of the 420 covariates, only antibiotic usage in the prior 6-12 weeks showed an independent 421 association with but gene quantity. Similarly, lower calculated relative abundance of the 422 but gene was significantly associated with ME/CFS status ( $\mathrm{FC}=0.60,95 \% \mathrm{Cl}: 0.39-0.91$, $423 p=0.017)$ in regression and only Hispanic race showed an independent association with 424 but gene relative abundance in the model (Supplementary Table 2).

425 These results confirm quantitative deficiency of the most important terminal gene 426 in the acetyl-CoA pathway of butyrate production in the feces of ME/CFS subjects 427 compared to healthy controls.

\section{Assessment of SCFAs in feces}

430 Given the strong evidence indicating reduced abundance and quantity of BPB and 431 reduced metagenomic capacity for producing butyrate, we measured SCFAs in all fecal 432 samples from ME/CFS and healthy control subjects using gas chromatography-mass 433 spectrometry. The fecal concentration of both acetate (Mann-Whitney $U$ test, $p=0.004$ ) 434 and butyrate (Mann-Whitney $U$ test, $p<0.0001$ ) were lower in ME/CFS compared to 435 healthy controls, while propionate was unchanged, in univariate analyses (Figure 3G). In 
436 stratified analyses by sr-IBS status, acetate was lower in ME/CFS subjects with sr-IBS

437 compared to healthy controls without sr-IBS (Mann-Whitney $U$ test, $p^{\text {adj }}=0.010$ ), but did

438 not differ in ME/CFS subjects without sr-IBS. In contrast, butyrate was lower in both

439 ME/CFS subjects without sr-IBS (Mann-Whitney $U$ test, $\mathrm{p}^{\text {adj }}<0.0001$ ) and with sr-IBS

$440 \quad$ (Mann-Whitney $U$ test, $\left.\mathrm{p}^{\text {adj }}=0.002\right)$ compared to healthy controls without sr-IBS (Figure $4413 \mathrm{H})$.

To further assess the relationships between quantitative measures of SCFAs in

443 feces (outcome variables) with ME/CFS status (main predictor), generalized linear 444 regression models with Gamma distribution with log link were fitted, adjusting for 445 covariates (Table 5). As with all our regression analyses, we evaluated the interaction 446 term between ME/CFS and sr-IBS. Unlike our prior regressions, the ME/CFS*sr-IBS 447 interaction term was significant. In order to better understand the nature of the 448 relationships among SCFA levels and ME/CFS and sr-IBS, we evaluated stratified 449 regression models, adjusted for covariates (bottom 3 rows in Table $\mathbf{5}$, and 450 Supplementary Tables 5A, 5B, 5C). Regression Model 2, comparing levels of SCFAs 451 between ME/CFS subjects without sr-IBS and healthy controls without sr-IBS, revealed 452 decreased quantities of both acetate ( $F C=0.87,95 \% \mathrm{Cl}: 0.77-0.98, p=0.022)$ and butyrate $453(\mathrm{FC}=0.60,95 \% \mathrm{Cl}: 0.48-0.75, \mathrm{p}<0.001)$, but not propionate $(\mathrm{FC}=0.90,95 \% \mathrm{Cl}: 0.78-1.04$, $454 \mathrm{p}=0.149$ ) in ME/CFS. Regression Model 3, comparing ME/CFS subjects with sr-IBS and 455 healthy controls without sr-IBS, revealed decreased quantities of acetate ( $F C=0.83,95 \%$ $456 \mathrm{Cl}: 0.71-0.96, \mathrm{p}=0.017)$, butyrate $(\mathrm{FC}=0.56,95 \% \mathrm{Cl}: 0.42-0.73, \mathrm{p}<0.001)$ and propionate $457(\mathrm{FC}=0.83,95 \% \mathrm{Cl}: 0.70-1.00, \mathrm{p}=0.047)$ in ME/CFS subjects with IBS. Regression Model 458 4, comparing ME/CFS subjects with sr-IBS and ME/CFS subjects without sr-IBS, did not 
459 identify differences in any of the SCFAs between these groups. These results indicate

460 that both deficient acetate and butyrate are associated with ME/CFS with or without

461 comorbid sr-IBS, while deficient propionate is only found in ME/CFS subjects with sr-IBS.

462 Further, there is a larger effect size in terms of the estimated fold change for fecal butyrate 463 concentrations compared with either acetate or propionate.

465 Relationships among measured variables and fatigue scores

466 Relative abundance, quantitative, and functional analyses of microbiota were consistent 467 and highly correlated even after FDR adjustment for Spearman's correlations (Figure 468 4A). Most measured factors showed positive correlations despite a wide range of 469 laboratory methods employed (metagenomics analysis of taxa relative abundance and 470 alpha diversity, metagenomics analysis of genes in the acetyl-CoA pathway, qPCR 471 analysis of bacterial taxa and the but gene, and GC-MS measures of stool SCFAs 472 including butyrate). Some of the stronger positive correlations such as those observed 473 between the relative abundance of $F$. prausnitzii determined by shotgun metagenomics 474 and the quantity and relative abundance of $F$. prausnitzii determined by qPCR, speak to 475 the reproducibility of our findings using different methods. Other positive correlations, 476 such as those among alpha diversity, gene counts along the acetyl-CoA pathway 477 (especially but) and the relative abundance or quantity of BPB may be more reflective of 478 biological links among diversity and function of bacteria. Positive correlations among 479 measured variables were generally reflective of measured variables that tended to be 480 deficient in ME/CFS or ME/CFS stratified by sr-IBS, which was the case for the majority 481 of measured variables evaluated here. Inverse correlations were generally restricted to 
482 relative abundance of bacterial taxa that were found to be enriched in ME/CFS 483 (Lachnoclostridium) or ME/CFS with sr-IBS (C. bolteae, F. plautii, Flavonifractor).

484 We also evaluated the relationship among our measured variables and fatigue 485 scores based on the five dimensions of the Multidimensional Fatigue Inventory (MFI) on 486 the whole dataset (all cases + all controls, $n=197$ ) (Figure 4B). In this case, the majority 487 of significant correlations among measured variables and fatigue scores after FDR 488 correction were inverse, as measured variables that tended to be deficient in ME/CFS 489 were associated with higher fatigue scores (more severe fatigue), especially for general 490 and physical fatigue and reduced activity. The relative abundance and qPCR quantitation 491 of bacterial species and the but gene, metagenomic content of genes along the acetyl492 CoA butyrate pathway, and levels of butyrate were inversely correlated with general 493 fatigue, physical fatigue and reduced activity.

494 As correlations in the whole dataset may reflect reduced levels of measured 495 variables and higher fatigue scores in individuals with ME/CFS vs. healthy controls 496 (Supplementary Table 6), we also evaluated associations between measured variables 497 and fatigue scores in ME/CFS subjects alone ( $n=106)$ (Figure 4C). Although fewer 498 correlations remained significant in the cases after FDR adjustment, various bacterial taxa 499 including F. prausnitzii, the genus Faecalibacterium, Roseburia inulinivorans, Roseburia 500 intestinalis, the genus Roseburia, and the genus Coprococcus correlated inversely with 501 either general fatigue, or physical fatigue, or both. The qPCR quantity for the but gene 502 correlated inversely with general fatigue and the qPCR quantity of $F$. prausnitzii in stool 503 correlated inversely with general fatigue, physical fatigue and reduced activity (Figure 
504 4C). Thus, the lower the abundance or quantity of these BPB, the more severe the fatigue 505 dimension scores in ME/CFS.

We also determined whether similar relationships existed among our measured

507 variables and fatigue scores in healthy control subjects alone $(n=91)$. In contrast to

508 ME/CFS subjects, no significant correlations were found in healthy controls after FDR

509 adjustment (as such, no correlogram is shown).

510 As sr-IBS could further influence these relationships, we evaluated the

511 relationships among measured variables and fatigue scores only in ME/CFS patients

512 without sr-IBS $(n=71)$ and only in ME/CFS patients with sr-IBS $(n=35)$. No significant

513 relationships were found for either stratified group after FDR adjustment (as such, no

514 correlograms are shown).

515 Overall, these findings suggest that deficiencies in BPB and their metabolic 516 pathways are correlated with the severity of fatigue symptoms, but these associations are 517 only found in ME/CFS, not healthy controls.

\section{Discussion}

520 Our analyses of the fecal microbiome in ME/CFS subjects and healthy controls 521 matched for age, sex, geography, and socioeconomic status indicated significant 522 differences in composition, function and metabolism. In a systematic review of studies on 523 gut dysbiosis in ME/CFS, Du Preez et al. concluded that intrinsic and extrinsic factors that 524 can influence microbiome composition should be better controlled for in case-control 525 studies [24]. One such factor that may be important in the context of ME/CFS and the 526 microbiome is IBS co-morbidity, which occurs at high prevalence in ME/CFS compared 
527 with healthy controls [31-33], and is a condition that is independently associated with

528 microbiome disturbances [35, 43, 44]. In our study, more ME/CFS patients reported

529 having an IBS diagnosis $(35 / 106 ; 33 \%)$ than healthy controls $(3 / 91 ; 3.3 \%)$. Both our

530 current and prior fecal shotgun metagenomic study support the notion that IBS co-

531 morbidity must be carefully considered in future ME/CFS microbiome studies. In fact,

532 differences in gut microbiome alpha diversity between ME/CFS subjects and healthy

533 controls appears to be largely driven by sr-IBS co-morbidity in ME/CFS, and individuals

534 with ME/CFS and sr-IBS had a range of distinct bacterial species with differential relative

535 abundance compared to healthy controls (i.e., A. putredinis, C. bolteae, F. plautii, D.

536 longicatena) that were not found when comparing ME/CFS without sr-IBS and healthy

537 controls. Thus, at least some microbiome differences in ME/CFS are confounded by IBS

538 co-morbidity. In contrast, microbiota beta diversity differed significantly between ME/CFS

539 and healthy controls; a difference that was independent of sr-IBS status.

541 Species and taxa linked to ME/CFS: Relative and Absolute Differences

542 GAMLSS-BEZI models, adjusted for important covariates (including sr-IBS),

543 identified differentially abundant fecal bacterial taxa between ME/CFS subjects and

544 healthy controls. These analyses identified two species (E. rectale and F. prausnitzii) and

545 six genera (Eubacterium, Faecalibacterium, Dorea, Roseburia, Gemmiger, and

546 Lachnoclostridium) that differed in relative abundance in ME/CFS compared to healthy

547 control subjects. Both the species E. rectale and F. prausnitzii and the genera

548 Eubacterium, Faecalibacterium and Roseburia are prominent BPB in the human GI tract

549 [41]. All had lower relative abundance in feces from ME/CFS subjects compared to 
550 healthy controls, independent of sr-IBS status. To test the validity of this finding, we 551 employed qPCR to quantitatively evaluate levels of Roseburia-Eubacterium and $F$. 552 prausnitzii. Both the fecal load of these BPB as well as their relative abundances were 553 deficient in fecal samples from ME/CFS patients.

554 An additional, unexpected and novel finding from our qPCR analyses was that 555 ME/CFS patients have higher total bacterial 16S rRNA genes/gram of feces than healthy 556 controls. Total fecal bacterial load is infrequently evaluated in microbiome studies and 557 little is still known about factors that may impact total bacterial load. However, it is well 558 documented that antibiotics can have a dramatic impact on bacterial load [45, 46]. In this 559 study, we controlled for antibiotics, both in our study design and statistical analyses. Thus, 560 it is unlikely that antibiotics can explain the differences in bacterial load.

561 Dietary factors that may impact bacterial load include low fermentable oligo-, di-, 562 mono-saccharides and polyols (FODMAPs) [47]. However, we cannot address this 563 possibility because detailed dietary information was not collected from subjects. While it 564 is possible that subject diets differ between ME/CFS subjects and controls, we note that 565 more ME/CFS subjects reported taking prebiotic fiber supplements $(11 / 106 ; 10.4 \%)$ than 566 healthy controls $(2 / 91 ; 2.2 \%)$. Prebiotic fibers typically stimulate the growth and activity of 567 BPB. The observation that ME/CFS subjects had lower levels of BPB and butyrate and 568 higher total bacterial load even with adjustment of regression models for prebiotic fiber 569 use, suggests that some factor, either associated with the pathobiology or symptoms of $570 \mathrm{ME} / \mathrm{CFS}$, rather than prebiotic fiber intake is selectively influencing these microbiome 571 changes. 
Other gastrointestinal disturbances such as severe acute malnutrition with acute

573 diarrhea and inflammatory bowel disease can also alter total bacterial load in feces or

574 intestinal mucosa, respectively $[48,49]$. However, subjects in this study were not acutely

575 malnourished and only two cases (2/106) reported a formal diagnosis of IBD; no controls

576 had a diagnosis of IBD. Small intestinal bacterial overgrowth is frequently associated with

577 functional gastrointestinal disorders such as IBS [50]. However, we are not aware of any

578 studies that have shown that fecal bacterial load is increased in IBS, and our findings

579 suggest that increased fecal bacterial load is associated with ME/CFS, independent of sr-

580 IBS. Further, despite the significant association of fecal bacterial load and ME/CFS, it

581 remains unclear whether higher fecal bacterial load is representative of bacterial

582 overgrowth in the intestine or a higher rate of bacterial washout or loss of adherent

583 mucosa-associated bacteria.

585 Specific butyrate-production deficiency in ME/CFS

586 Our functional metagenomic analysis found that the overall bacterial capacity for 587 butyrate production is deficient in ME/CFS. To delve deeper into the specific pathways of 588 butyrate production that are deficient, we examined the bacterial gene content of the four 589 bacterial butyrate production pathways. Only the gene content for the acetyl-CoA pathway 590 was deficient in ME/CFS. The acetyl-CoA pathway is the dominant pathway of butyrate 591 production in the human gut. Whereas this pathway for butyrate production is fueled by 592 carbohydrates, the other three pathways (glutarate, lysine and 4-aminobutyrate 593 pathways) are fueled by proteins [41]. Counts per million of genes in the acetyl-CoA 594 pathway were substantially higher than for genes in the other three pathways. 
The majority of bacteria that utilize the acetyl-CoA pathway typically encode either

596 the but gene or the buk gene to complete the terminal reaction of butyrate production.

597 The but gene is dominant in the human gut [41]. We found that whereas the but gene was

598 deficient in ME/CFS, the buk gene was not. Several of the bacteria that we found at lower

599 relative abundance and quantity in ME/CFS, including F. prausnitzii, E. rectale, Roseburia

600 spp. and Eubacterium spp. are known to encode the genes for the acetyl-CoA pathway

601 of butyrate [41]. Our functional metagenomic analyses showed that ME/CFS subjects are

602 specifically deficient in BPB that rely on the terminal but gene to produce butyrate. QPCR

603 and metabolomic analyses corroborated our functional metagenomic findings by

604 confirming that the quantity of the bacterially encoded but genes and levels of butyrate

605 are lower in feces of ME/CFS patients than in healthy controls.

In addition to butyrate, ME/CFS subjects have reduced quantities of fecal acetate,

607 although the degree of deficiency is less substantial than that of butyrate. Metabolic cross-

608 feeding interactions between bacterial groups are likely important determinants of the

609 composition of the intestinal microbial community. Acetate produced by bacterial

610 fermentation of carbohydrates or acetogens is utilized by BPB (those using the acetyl-

611 CoA pathway) for butyrate production, and BPB like F. prausnitzii, Roseburia spp. and

612 Eubacterium spp. may grow poorly in the absence of acetate [51-53]. Thus, net acetate

613 deficiency may contribute to deficient BPBs and butyrate and may be associated with

614 known acetate producers such as the genera Dorea and Fusicatenibacter, which were

615 also lower in relative abundance in ME/CFS compared to healthy controls $[54,55]$. In

616 contrast to butyrate and acetate, propionate was only reduced in patients with sr-IBS. 
617 Prior research has shown decreased concentration of fecal propionate in constipation618 predominant IBS $[56,57]$.

619 Butyrate is an important health-promoting bacterial metabolite in the GI tract with 620 diverse beneficial properties, including its role in host energy metabolism. Butyrate serves 621 as the primary energy source for colonocytes, accounting for $70 \%$ of the energy obtained 622 by epithelial cells in the colon [58]. In addition, butyrate influences proliferation of intestinal 623 epithelial and stem/progenitor cells, suppresses cancer cell proliferation, and can

624 influence epigenetic changes as a histone deacetylase inhibitor. Butyrate also plays a 625 role in promoting epithelial barrier function by regulating HIF-1 and tight junction proteins $626[59]$.

627 Finally, butyrate also mediates important immunomodulatory functions in the 628 intestine by promoting regulatory T cells, inhibiting inflammatory cytokine production, and 629 inducing antimicrobial activity in macrophages [60].

630 Thus, deficiency in this intestinal homeostatic metabolite could contribute to a 631 range of detrimental physiological disturbances including a weakened epithelial barrier 632 and enhanced intestinal inflammation. Evidence for disruption of intestinal barrier function 633 is supported by prior research showing elevated levels of plasma lipopolysaccharides in $634 \mathrm{ME} / \mathrm{CFS}$, indicative of microbial translocation [28].

\section{Correlation between butyrate deficiency and symptoms}

The relative abundance and/or quantity of fecal BPB are inversely correlated with

638 magnitude of symptoms, as reflected by the Multidimensional Fatigue Inventory, in 639 ME/CFS subjects. The magnitude of general fatigue and/or physical fatigue was greater 
640 in ME/CFS subjects that had lower relative abundance or quantity of $F$. prausnitzii, 641 Roseburia spp., Ruminococcus, and Coprococcus. The quantity of fecal F. prausnitzii

642 based on qPCR was inversely correlated with general fatigue, physical fatigue and 643 reduced activity. F. prausnitzii is an important member of the human microbiota, 644 representing $5 \%$ of the microbiota in healthy adults. The functional importance of $F$. 645 prausnitzii in the intestine may extend beyond its role as a BPB to include additional anti646 inflammatory effects through its production of microbial anti-inflammatory molecule 647 (MAM) as well as salicylic acid. As a potential biosensor of human health, deficiency of 648 fecal F. prausnitzii has been associated with a range of other conditions including IBD, 649 IBS, celiac disease, colorectal cancer, obesity, and more recently in patients during and 650 even after recovery from infection with SARS-CoV-2 [61-67]. In a large cohort, the 651 Flemish Gut Flora Project, Faecalibacterium along with Coprococcus were associated 652 with higher quality of life scores [68]. Patients with IBD and fatigue have reduced levels 653 of fecal F. prausnitzii compared with IBD patients without fatigue, further supporting the 654 association of this bacterium with fatigue symptoms [69].

655 The debilitating fatigue experienced by people with ME/CFS reduces physical 656 activity to a level substantially lower than seen in healthy controls [70-72]. In fact, 657 individuals with ME/CFS may engage in less high-intensity physical activity than even 658 sedentary control subjects [73]. In recent years, it has become clear from numerous 659 studies that physical activity/exercise has a substantive effect on the composition and 660 function of the gut microbiome. A theme is emerging in the field of exercise research 661 based on numerous studies in laboratory animals and humans that suggests that physical 662 activity influences the microbiome, stimulates BPB (especially Faecalibacterium and 
663 Roseburia species), and ultimately increases the levels of SCFAs [74, 75]. The 664 mechanisms by which physical activity may influence the microbiome are not fully 665 elucidated but such mechanisms may relate to the impact of exercise on blood flow to the 666 intestine, gut barrier integrity, transit time in the large intestine, enterohepatic circulation 667 of bile acids, contraction of skeletal muscles and metabolic flux, or raising of core body 668 temperature [74, 75]. Given the debilitating nature of ME/CFS, we acknowledge that the 669 deficiency in butyrate and BPB may arise as a result of the symptoms of ME/CFS (i.e.,

670 fatigue, post-exertional malaise, pain) and the behavioral adjustment to those symptoms

671 (i.e., reduced physical activity). Nonetheless, even if deficient butyrate-producing capacity

672 is a consequence rather than a direct cause of symptoms, such a deficiency could both

673 exacerbate ME/CFS-specific symptoms or potentiate the risk for the development of 674 additional health-related issues.

675

676 Conclusions

677 Given the importance of butyrate in preventing inflammation, fortifying the intestinal 678 barrier and promoting overall human health, the potential pathophysiological implications 679 of butyrate deficiency in ME/CFS warrant further investigation and may provide an 680 important target for treatment through prebiotic, probiotic or synbiotic interventions aimed 681 at boosting butyrate production in the intestine. 


\section{Methods}

\section{Study Population}

688 The initial cohort consisted of $177 \mathrm{ME} / \mathrm{CFS}$ cases and 177 healthy controls prescreened 689 at five geographically-diverse ME/CFS clinics across the USA (Incline Village, NV; Miami, 690 FL; New York, NY; Palo Alto, CA; Salt Lake City, UT) as part of a National Institutes of 691 Health-sponsored R56 study. ME/CFS cases met the requirements of both the 1994 CDC 692 [1] and the 2003 Canadian consensus criteria [9] for ME/CFS. Control participants were 693 matched to ME/CFS cases based on geographical/clinical site, sex, age, race/ethnicity, 694 and date of sampling ( \pm 30 days $)$.

The CDC Criteria require that cases have fatigue persisting for greater than six 696 months that is clinically-evaluated, persistent or relapsing, and which meets five criteria: 697 is of new onset, is not the result of ongoing exertion, is not alleviated by rest, is made 698 worse by exertion, and results in substantial reduction in previous levels of activity. The 699 CDC criteria additionally require the concurrent occurrence of at least four of the following 700 symptoms for at least six consecutive months: sore throat, tender cervical or axillary 701 lymph nodes, muscle pain, multiple joint pain without swelling or redness, headaches of 702 new or different type, unrefreshing sleep, post-exertional malaise, and impaired memory 703 or concentration. The Canadian consensus criteria impose additional restrictions, 704 requiring at least two neurologic/cognitive manifestations, and at least one clinical feature 705 from two of the following three categories: autonomic manifestations, neuroendocrine 706 manifestations, and immune manifestations.

707 Eligible cases must also have had a diminished or restricted capacity to work, 708 reported a viral-like prodrome prior to onset of ME/CFS, and met a low-score threshold 
709 for two out of the following three domains measured by the self-reported Short Form-36

710 General Health Survey (SF-36): vitality $<35$, social functioning $<62.5$, role-physical $<50$.

711 Additional exclusion criteria for both cases and controls included disorders or treatments

712 resulting in immunosuppression, and antibiotic use within six weeks prior to the baseline

713 assessment. Based on these screening criteria, we excluded five ME/CFS cases and one

714 control participant prior to the baseline assessment.

715 In the current study, a nested sub-cohort was established for participants with

716 complete survey data collection and biospecimen (stool) collection at the first and fourth

717 (final) timepoints for the overall study. Participants were frequency-matched on key

718 demographic elements to ensure similarity between the nested cohort and full cohort.

719 This sub-cohort is comprised of $106 \mathrm{ME} / \mathrm{CFS}$ cases and 91 healthy controls that met

720 these criteria; the derivation of this sub-cohort is outlined in Supplementary Figure 6. All

721 subjects provided written consent in accordance with study protocols approved by the

722 Columbia University Medical Center Institutional Review Board (IRB).

723

\section{Clinical Assessments}

725 All subjects completed standardized screening instruments to assess medical history,

726 family medical history, current medication use, symptom scores, and

727 demographic/lifestyle information, as well as a baseline SF-36. All subjects underwent a

728 screening blood draw to determine that they had normal values in the following three

729 laboratory tests from Quest Diagnostics: complete blood count with differential,

730 comprehensive metabolic panel, thyroid stimulating hormone (TSH). 
Subjects eligible for participation after the baseline visit returned to the same clinic

732 four times over the course of one year for sample collection and completion of survey

733 instruments. At each of the four visits subjects completed a range of rating scales,

734 including the Multidimensional Fatigue Inventory (MFI). Prior to all four study visits,

735 subjects were provided with at-home collection kits for stool. Stool samples were collected

736 within 48 hours prior to each study visit and refrigerated until the day of the visit. All

737 samples were shipped to the Columbia University laboratory site in insulated Styrofoam

738 boxes with frozen and refrigerated gel packs. The stool samples were aliquoted, weighed

739 and moved to $-80{ }^{\circ} \mathrm{C}$ freezers for storage. Only samples and clinical rating scale data

740 obtained at the first study visit were analyzed as part of the present study.

741 On the medical history questionnaire, participants were asked to self-report if they

742 received an IBS diagnosis (sr-IBS) by a physician and the date of diagnosis. In the

743 analytic sub-cohort, 35 out of the 106 (33.0\%) ME/CFS cases reported sr-IBS, while only

7443 out of the 91 control subjects (3.3\%) reported sr-IBS. The use of probiotic and prebiotic

745 supplements was specifically included in the "current medication use" data collection

746 instrument, which determined the frequency and recent use of these products. Any

747 subjects that indicated consumption of these supplements daily or a few times a week

748 and reported using them within the last week prior to their study visit would be endorsed

749 for that type of supplement. Participants also reported any antibiotic use that occurred

750 outside of the six-week window for study eligibility.

751 The MFI consists of a 20-item self-reported questionnaire that evaluates five

752 dimensions of fatigue: general, physical and mental fatigue, reduced activity, and reduced

753 motivation [76]. The scoring for this instrument was transformed into a $0-100$ scale to 
754 allow for comparisons between dimensions: a score of 100 was equivalent to maximum

755 disability or severity and a score of zero was equivalent to no disability or disturbance.

\section{Fecal DNA Extraction}

758 A modified protocol of the QIAmp DNA Stool Mini Kit (Qiagen Inc; Valencia CA, USA) 759 was used for the extraction of DNA from stool samples. Prior to the initiation of the 760 extraction process, all disposable elements including tubes, columns, $0.1 \mathrm{~mm}$ and $0.5 \mathrm{~mm}$ 761 glass beads (MoBio Laboratories) were UV irradiated twice at a distance of 1 inch from 762 UV bulbs and at $3000 \times 100 \mu \mathrm{J} / \mathrm{cm}^{2}$ in a SpectroLinker XL-1500 UV crosslinker

763 (Spectronics Corporation). All liquid extraction reagents in the kit were aliquoted into 2 $764 \mathrm{~mL}$ tubes in a UV hood at $\leq 1 \mathrm{ml}$ and UV irradiated as mentioned above. To confirm that 765 bacterial $16 \mathrm{~S}$ rDNA contaminants were not introduced from the extraction reagents, two 766 empty $2 \mathrm{~mL}$ tubes were used for each set of DNA extraction to serve as negative controls 767 throughout the entire process. Each fecal sample was weighed and $<220 \mathrm{mg}$ of each 768 fecal sample was re-suspended in AL Buffer (Qiagen). Glass beads $(0.1 \mathrm{~mm}$ and $0.5 \mathrm{~mm}$, 769 MoBio Laboratories) were added to the mixture. To ensure Gram-positive bacteria lysis, 770 samples were disrupted by bead beating in a TissueLyser (Qiagen) for 5 minutes at $30 \mathrm{~Hz}$ 771 and incubated for 5 minutes at $95^{\circ} \mathrm{C}$. The remaining steps for DNA extraction were 772 performed in a UV hood by following the manufacturer's protocol. To determine DNA 773 concentration and purity, a NanoDrop ND-100 spectrophotometer (NanoDrop 774 Technnologies, Wilmington, DE) was used and the extracted DNA samples were stored 775 at $-80^{\circ} \mathrm{C}$. 


\section{Shotgun metagenomic sequencing and bioinformatic analyses}

778 Shot-gun metagenomics sequencing was carried out on DNA extracts obtained from 197

779 fecal samples (106 ME/CFS cases and 91 healthy controls). For Illumina library 780 preparation, genomic DNA was sheared to a 200-bp average fragment length using a 781 Covaris E210 focused ultrasonicator. Sheared DNA was purified and used for Illumina 782 library construction using the KAPA Hyper Prep kit (KK8504, Kapa Biosystems).

783 Sequencing libraries were quantified using an Agilent Bioanalyzer 2100. Sequencing was 784 carried out on the Illumina HiSeq 4000 platform (Illumina, San Diego, CA, USA). 785 Sequencing libraries from samples of cases and controls were grouped into eight different 786 sequencing pools. Each sample yielded $27.8 \pm 10.3$ (mean \pm sd) million reads. The 787 demultiplexed raw FastQ files were adapter trimmed using Cutadapt [77]. Adaptor 788 trimming was followed by the generation of quality reports using FastQC and filtering with 789 PrinSEQ [78]. Host background levels were determined by mapping the filtered reads 790 against the human genome using Bowtie2 mapper [79]. After the step of host subtraction, $79125.1 \pm 9.0$ (mean \pm sd) million reads per sample remained on average. Non-host reads 792 were subjected to Kraken2 for taxonomy classification [80]. Kraken2 matches each K793 mer within a query sequence to the lowest common ancestor (LCA) of all genomes in the 794 database containing the given K-mer. Our Kraken2 local database included all 16,799

795 fully sequenced and representative bacteria species genomes in the RefSeq database 796 (December 2018). The species-level taxonomy abundances were estimated using 797 Bracken, which is recommended to perform a Bayesian estimation of taxonomy 798 abundance after the use of Kraken2 [80, 81]. Structural zeros in the abundance table 799 were further identified using the program Analysis of Microbiome Data in the Presence of 
800 Excess Zeros version II (ANCOM-II) [82]. The three groups we have compared in our 801 study include ME/CFS with sr-IBS, ME/CFS without sr-IBS, and healthy controls. The

802 taxa presenting as structural zeros in all three categories were eliminated from the 803 dataset. The data was rarefied prior to diversity analyses using a depth of 500,000 reads.

804 Diversity metrics (alpha diversity: Shannon index, pielou's evenness, and observed otus;

805 beta-diversity: Bray-Curtis dissimilarity) were calculated and plotted using the core806 diversity plugin and the emperor plugin within QIIME2 [83]. The beta diversity significance

807 among groups was examined by QIIME2 diversity plugin with PERMANOVA tests. To 808 evaluate fecal metabolic functional profiles from SMS data, KEGG gene family 809 abundances and the functional pathway and module abundances were calculated using 810 FMAP with the default database [84]. The abundance of genes involved in butyrate 811 synthesis was calculated by aligning host subtracted sequence reads to a curated 812 database specifically consisting of butyrate synthesis pathway related genes [41], using 813 customized scripts from the FMAP pipeline.

\section{5 qPCR}

816 Plasmid DNA standards were constructed using published qPCR primers targeting the 817 bacterial $16 \mathrm{~S}$ rRNA gene of Faecalibacterium prausnitzii and Eubacterium/Roseburia 818 genera [85], the bacterial but gene $(\mathrm{BcoA})$ [42], and broad range bacterial 16S rRNA 819 genes to quantitate total bacteria [86] for absolute quantitation. Bacterial gene targets 820 were first amplified by conventional PCR from human stool samples. PCR products were 821 run on $1 \%$ agarose gels and purified using the QIAgen Gel Extraction kit. Purified products 822 were ligated into pGEM T-Easy vector (Promega), transformed into DH5 $\alpha$ competent cells, 
823 and cultured on Luria Bertani plates with ampicillin. Colonies were inoculated into Luria

824 broth with ampicillin $(5 \mathrm{ml})$, and plasmids were extracted with Pure Link Plasmid

825 Extraction Kit (Invitrogen). After verifying that plasmid sequences had 100\% nucleotide

826 similarity to the target bacterial taxa or the bacterial but gene, plasmids were linearized

827 with the restriction enzyme Sphl, and 10-fold serial dilutions were generated ranging from

$8285 \times 10^{6}$ to 5 copies (note for Eubacterium/Roseburia standards, two plasmids were

829 combined consisting of one clone matching Eubacterium hallii and one clone matching

830 Roseburia hominis). Salmon sperm DNA at $2.5 \mathrm{ng} / \mu \mathrm{l}$ was spiked into the standard

831 dilutions as background DNA. Real time PCR was performed with the ABI StepOne Plus

832 Real Time PCR system (Applied Biosystems). Amplification plots for all plasmid

833 standards were sensitive down to five copies and standard curves generated yielded

834 correlation coefficients ranging from 0.998 to 1 . For probe-based assays (total bacteria),

835 each $25 \mu \mathrm{L}$ real time PCR reaction consisted of $1 \mathrm{x}$ TaqMan Universal PCR Master Mix

836 (Applied Biosystems), $300 \mathrm{nM}$ primers, $200 \mathrm{nM}$ probe and $5 \mathrm{ng}$ of fecal DNA. The total

837 bacteria probe was labeled with a 5'-end fluorescent reporter (6-carboxyfluorescine, FAM)

838 and had a 3'-end black hole quencher (BHQ1a, Operon). For SYBR green-based assays,

839 each $25 \mu$ reaction consisted of 1X SYBR Green PCR master mix (Applied biosystems),

$840300 \mathrm{nM}$ primers and $5 \mathrm{ng}$ of fecal DNA. Cycling conditions consisted of $50^{\circ} \mathrm{C}$ for $2 \mathrm{~min}$,

$84195^{\circ} \mathrm{C}$ for $10 \mathrm{~min}$, and $45 \mathrm{cycles}$ at $95^{\circ} \mathrm{C}$ for $15 \mathrm{~s}$ and $60^{\circ} \mathrm{C}$ for $1 \mathrm{~min} 30 \mathrm{~s}$ (for total bacteria,

842 Eubacterium/Roseburia 16s and Faecalibacterium 16s assays). For but gene the cycle

843 conditions were $50^{\circ} \mathrm{C}$ for $2 \mathrm{~min}, 95^{\circ} \mathrm{C}$ for $10 \mathrm{~min}$, and 45 cycles at $95^{\circ} \mathrm{C}$ for $15 \mathrm{~s}, 53^{\circ} \mathrm{C}$

844 for $1 \mathrm{~min} 45 \mathrm{~s}$ and $77^{\circ} \mathrm{C}$ for $30 \mathrm{~s}$ (data collection). All samples were run in duplicate and 845 averaged for each assay. Absolute quantity was determined based on the weight of fecal 
846 samples and expressed as copy numbers per gram of feces. The calculated relative

847 abundances of bacterial taxa and the but gene were also assessed by dividing the

848 absolute copy number for the targeted bacteria (F. prausnitzii or Eubacterium/Roseburia)

849 or but gene by the total bacterial $16 \mathrm{~S}$ copy number.

\section{Fecal SCFA metabolomics}

852 Fecal SCFAs were measured by gas chromatography-mass spectrometry (GC-MS) as 853 previously described [87] with minor modifications. Fecal samples were homogenized and $85410 \mathrm{mg}$ resuspended in $0.5 \mathrm{ml}$ 2-ethylbutyric acid $(100 \mu \mathrm{g} / \mathrm{ml}$; as internal standard 1$)$ in 855 LC/MS grade water. The sample was acidified with $0.1 \mathrm{ml}$ concentrated hydrochloric acid, 856 vortexed and placed on ice. $1 \mathrm{ml}$ of 4 -methylvaleric acid $(100 \mu \mathrm{g} / \mathrm{ml}$; as internal standard 857 2) in tert-butyl methyl ether (MTBE) was added, the sample was vortexed and shaken for $85830 \mathrm{~min}$ at room temperature, and centrifuged for $2 \mathrm{~min}$ at $14,000 \mathrm{rcf}$. Supernatant was 859 collected after centrifugation and $0.1 \mathrm{~g}$ of anhydrous sodium sulfate was added to it, 860 vortexed and incubated at room temperature for $10 \mathrm{~min}$. Finally, $100 \mu \mathrm{l}$ of the supernatant 861 was combined with $25 \mu \mathrm{l} \quad \mathrm{N}$-tert-Butyldimethylsilyl-N-methyltrifluoroacetamide

862 (MTBSTFA). Calibration standards containing acetic acid, n-butyric acid, and propionic 863 acid as well as the internal standards, 2-ethylbutyric acid and 4-methylvaleric acid, were 864 prepared and derivatized with MTBSTFA. After adding MTBSTFA as a derivatization 865 reagent and incubating for $30 \mathrm{~min}$ at $80^{\circ} \mathrm{C}$ followed by 1 hour at room temperature, 866 samples were injected with a 100:1 split into an Agilent GC7890B gas chromatograph 867 coupled with an Agilent 5977MS mass spectrometer detector. Helium was used as a 868 carrier gas. Analyses were performed using a DB5 duraguard capillary column (30 m x 
$8690.25 \mathrm{~mm} \times 0.25 \mu \mathrm{m}$ film thickness). The column head pressure was 9.83 psi. Injector, 870 source and quadrupole temperatures were $250^{\circ} \mathrm{C}, 290^{\circ} \mathrm{C}, 150^{\circ} \mathrm{C}$, respectively. The GC

871 oven program was $50^{\circ} \mathrm{C}$ for $0.5 \mathrm{~min}$, increased to $70^{\circ} \mathrm{C}$ for $3.5 \mathrm{~min}$ at $5^{\circ} \mathrm{C} / \mathrm{min}$, increased 872 to $120^{\circ} \mathrm{C}$ at $10^{\circ} \mathrm{C} / \mathrm{min}$, and increased to $290^{\circ} \mathrm{C}$ for $3 \mathrm{~min}$ at $35^{\circ} \mathrm{C} / \mathrm{min}$. The total run time 873 was $20.857 \mathrm{~min}$.

\section{Statistical analyses}

876 Cohort characteristics: Differences in cohort characteristics between ME/CFS and 877 healthy control subjects were assessed using the Mann-Whitney $U$ test for continuous 878 variables and either the Fisher's Exact test or Chi-squared test for categorical variables 879 and $p<0.05$ was considered statistically significant.

881 Box-and-whiskers plots, Mann-Whitney U-test and Kruskal-Wallis test: All box-and882 whiskers plots shown in the main and supplementary figures represent the interquartile 883 ranges (25th through 75th percentiles, boxes), medians (50th percentiles, bars within the 884 boxes), the 5th and 95th percentiles (whiskers above and below the boxes), and outliers 885 beyond the whiskers (closed circles). Plots were created and statistical analyses 886 performed using Prism 7 (GraphPad Software, CA). All statistics based on data presented 887 in box-and-whiskers plots comparing all ME/CFS subjects and healthy controls are two888 tailed $P$-values derived from Mann-Whitney $U$-tests. For stratified analyses of more than 889 two groups, significance was first determined based on the Kruskal-Wallis test. If the 890 Kruskal-Wallis test was significant at $p<0.05$, then between group significance was 
891 determined based on the Mann-Whitney $U$ test with multiple testing (Bonferroni)

892 correction applied, and an adjusted $p$-value $\left(p^{\text {adj }}\right)<0.05$ was considered significant.

893

894 Beta diversity: Beta diversity was assessed based on the Bray-Curtis dissimilarity metric.

895 Differences in beta diversity using Principal coordinate analyses (PCoA) were visualized

896 with 3-D plots using QIIME2. Permutational multivariate analysis of variance

897 (PERMANOVA) with 999 Monte Carlo permutations was used to evaluate the statistical

898 significance of the dissimilarity between groups. The Freedman-Lane PERMANOVA test

899 [88] was used to assess differences in microbiota beta diversity between ME/CFS and

900 healthy controls, and was adjusted for covariates (sr-IBS status, site of sampling, sex,

901 BMI, race/ethnicity, age, antibiotic usage 6-12 weeks prior to sample collection, probiotic

902 supplement use, and prebiotic supplement use). Two-group comparisons were

903 considered significant at $p<0.05$. For stratified analyses (more than two groups), the

904 FDR was controlled by Bonferroni correction and an adjusted $p$-value $\left(p^{\text {adj }}\right)<0.05$ was

905 considered significant.

906

907 Differential Taxa Abundance, GAMLSS-BEZI models: Prior to differential abundance 908 analysis, PERFect:Permutation was used to remove taxa that are present due to 909 contamination or otherwise are unrelated to ME/CFS [89]. The regression then used to 910 analyze the binary outcome ME/CFS was a Generalized Additive Model for Location,

911 Scale and Shape with a zero-inflated beta distribution (GAMLSS-BEZI) performed using

912 the metamicrobiomeR package [39]. This package was built in order to deal with zero 913 inflated, compositional data such as microbiome relative abundance. Features in the 
914 abundance table were pre-filtered, using the built-in pre-filtering step in

915 metamicrobiomeR, to remove features with mean relative abundance $\leq 0.1 \%$ and with

916 prevalence $\leq 5 \%$ across samples. With GAMLSS-BEZI a multivariate regression was

917 performed with ME/CFS status as the variable for comparison and adjusted for covariates

918 (sr-IBS diagnosis, site of recruitment, sex, BMI, race and ethnicity, age, antibiotic use

919 within 6-12 weeks of testing, probiotic supplement use and prebiotic supplement use) for

920 each taxon (species and genera were assessed separately) present in the data. Use of

921 prescription narcotics and antidepressants were tested in each model but did not affect

922 the results and were therefore not included in the final models. Multi-level categorical

923 variables were assigned as dummy variables and continuous variables were scaled using

924 z-scores. The original dataset includes 197 observations, 106 cases and 91 controls. Four

925 models were assessed, Model 1: comparing ME/CFS vs. healthy controls, Model 2:

926 ME/CFS without sr-IBS vs. healthy controls without sr-IBS, Model 3: ME/CFS with sr-IBS

927 vs. healthy controls without sr-IBS, and Model 4: ME/CFS with sr-IBS vs. ME/CFS without

928 sr-IBS. The $p$ values were adjusted for multiple comparisons using Benjamini-Hochberg

929 false discovery rate (FDR) procedure, and adjusted $p$ value $<0.05$ was considered

930 statistically significant. The odds ratio and $95 \%$ confidence intervals are used to interpret

931 the model.

932

933 Differential gut bacterial metabolic pathway modules: KEGG ontology profiles, 934 determined using FMAP, were assigned to gut metabolic module profiles using GOmixer

935 [40]. Analyses focused on the top 2000 most abundant bacterial KEGG orthologs. 936 Statistically over/under-represented gut metabolic modules between ME/CFS and 
937 controls were determined using GOmixer, which applies a Wilcoxon rank-sum test and

938 the Benjamini-Hochberg false discovery rate (FDR) to correct for multiple testing. Data

939 were scaled and the mean differences in pathways and modules were considered

940 significant at an FDR adjusted p-value $<0.1$.

942 Regression analyses: Fecal bacterial alpha diversity, qPCR quantities of fecal bacteria 943 and the but gene, fecal SCFAs concentrations and metagenomic content of genes

944 involved in butyrate synthesis pathways were all assessed with generalized linear 945 regressions. All models were adjusted for covariates (sr-IBS diagnosis, testing site, sex,

946 BMI, race and ethnicity, age, antibiotic use within 6-12 weeks of testing, probiotic

947 supplement use and prebiotic supplement use). Use of prescription narcotics and 948 antidepressants were tested in each model but did not affect the results and were 949 therefore not included in the final models. Data distribution was evaluated using 950 histograms for each outcome, and a variety of linear models were chosen after model fit 951 was tested utilizing the BIC measure. For count data a negative binomial and Poisson 952 regression were evaluated, for continuous data with a correct assumption of a normal 953 distribution a linear regression was used, and for continuous data with a non-normal 954 distribution a generalized linear regression with Gamma distribution with log link model 955 was compared with a lognormal generalized linear regression model. The SCFA 956 concentrations were assessed with a generalized linear regression with Gamma 957 distribution with log link. The alpha diversity measures of Shannon index and Evenness 958 were log transformed and were evaluated with a linear regression assuming normal 959 distribution. The observed species measure was assessed using a negative binomial 
960 generalized linear model. The metagenomic content for each butyrate-producing gene in

961 counts per million (CPM) was estimated with a generalized linear regression with Gamma

962 distribution with log link. Finally, the RT-qPCR data in copies/gram feces was evaluated

963 with a generalized linear regression with Gamma distribution with log link. For the

964 generalized linear models with Gamma distribution with log link and the negative binomial

965 generalized linear model, the exponent of the estimate is the fold change, and the 966 coefficients of linear regressions assuming normal distribution indicates the linearity of

967 the association between the predictors and outcome. For each regression an interaction

968 term between ME/CFS and sr-IBS was tested to determine if the relationship between

969 ME/CFS and the outcome was altered significantly by sr-IBS status. When the interaction

970 term was not significant, it was removed from the final models. The interaction term was

971 only significant for SCFAs models, and is shown in the result. Stratified analyses were

972 conducted to evaluate the nature of the interaction in these models. Results were 973 considered significant for predictor variables for all regression models when $p$-value < 9740.05.

975

976 Correlations: Correlations among measured variables (alpha diversity, relative

977 abundance of bacterial taxa, qPCR quantity of bacterial taxa and the bacterial but gene, 978 metagenomic content for genes along the acetyl-CoA pathway of butyrate production, 979 and the concentration of fecal SCFAs) and between measured variables and the scores 980 for the five dimensions (General Fatigue, Physical Fatigue, Mental Fatigue, Reduced 981 Activity, and Reduced Motivation) of the Multidimensional Fatigue Inventory (MFI) were 982 examined using nonparametric Spearman's correlation. FDR was controlled with the 
983 Benjamini-Hochberg procedure. Correlations with adjusted p-values $<0.05$ were

984 considered significant. Correlograms were created using the corrplot package in R.

985

986 Declarations

987 Ethics approval and consent to participate

988 All subjects provided written consent in accordance with study protocols approved by the

989 Columbia University Medical Center Institutional Review Board (IRB).

990

991 Consent for publication

992 Not applicable.

993

994 Availability of data and material

995 Host subtracted, shotgun metagenomic sequences from fecal samples of all ME/CFS and 996 healthy control samples are available from the Sequence Read Archive (SRA) under 997 PRJNA751448. All additional relevant data are available in this article and its 998 Supplementary Information files, or from the corresponding author upon request.

999

1000 Competing interests

1001 The authors declare that they have no competing interests.

1002

1003 Funding

1004 This study was made possible by the National Institutes of Health grant to the Center for

1005 Solutions for ME/CFS at Columbia University (grant No. 1U54AI138370). 
1007 Author Contributions

1008 WIL, TB, AC, DM, MH, ALK, SL, LB, SDV, NGK, JGM, DLP, and BLW contributed to 1009 study design. WIL, TB, OA, AR, and BLW designed the experiments. CG, XC, OA, RAY, $1010 \mathrm{AC}, \mathrm{AR}$, and BLW designed analyses. CG, XC, OA, RAY, AR, and BLW analyzed the 1011 data. CG, XC, RAY, AC and BLW wrote the paper. All authors reviewed and edited the 1012 manuscript.

1013

1014 Acknowledgements

1015 Not applicable

1016

1017

1018

1019

1020

1021

1022

1023

1024

1025

1026

1027

1028 

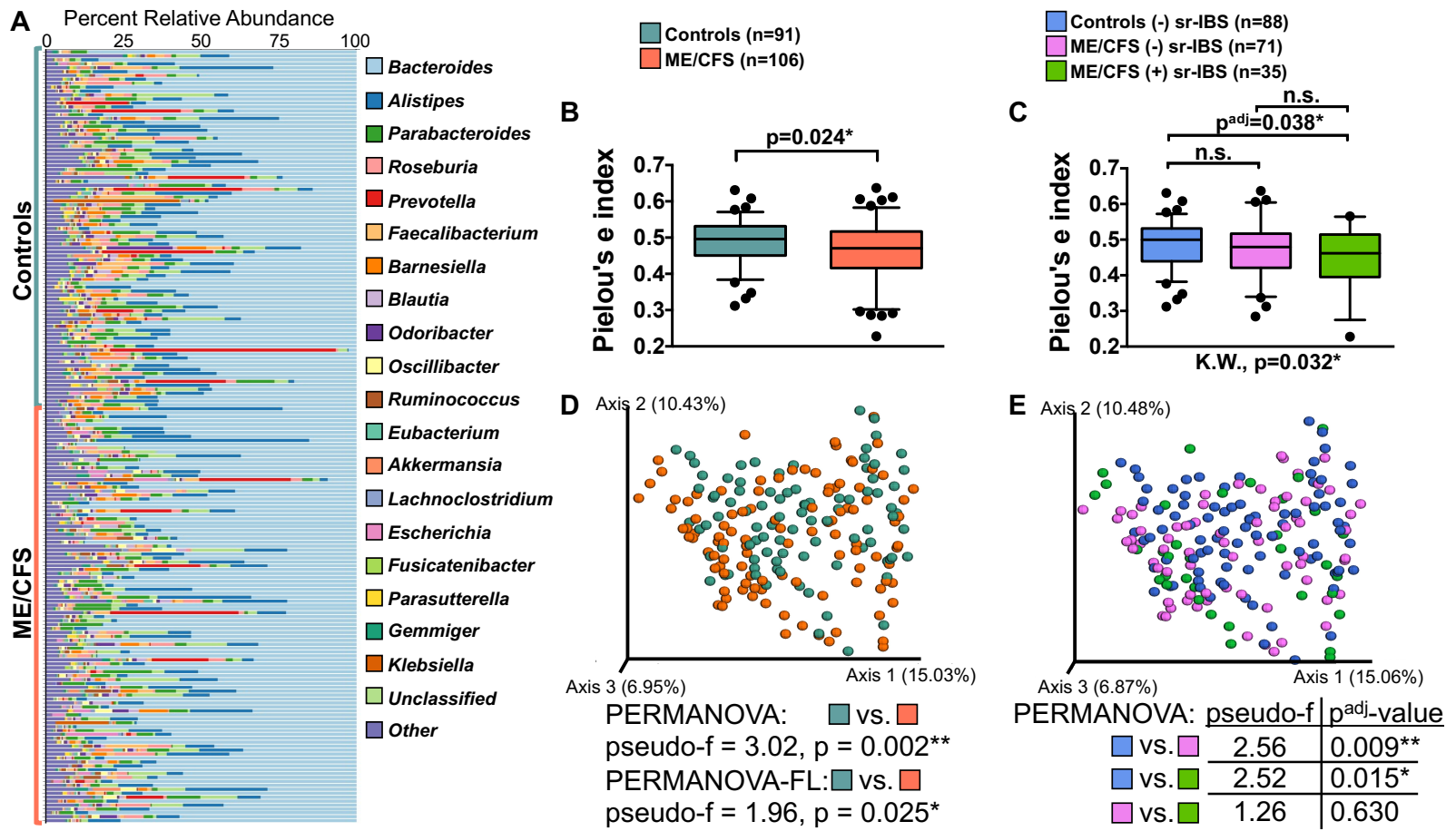

1031 Figure 1: Gut microbiome diversity. Stacked bar chart showing the percent relative abundance of the most abundant bacterial genera in ME/CFS ( $n=106)$ and healthy control subjects $(n=91)(A)$. Box-and-whiskers plots showing the distribution of microbiome alpha diversity based on Pielou's e index (evenness) between ME/CFS and healthy controls (B) and among stratified groups for healthy controls without (-) sr-IBS ( $n=88)$, ME/CFS subjects without $(-)$ sr-IBS $(n=71)$, and ME/CFS subjects with $(+)$ sr-IBS $(n=35)(C)$. Statistical significance was determined based on two-tailed $p$-values from the MannWhitney $U$ test (B). For stratified analyses significance was first determined based on the Kruskal-Wallis test (K.W., results shown below figure in $\mathbf{C}$ ) and between group significance was determined based on the Mann-Whitney $U$ test with multiple testing

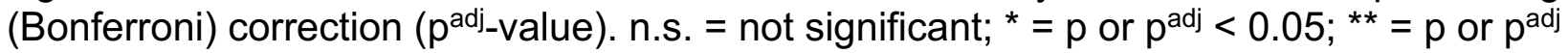
$<0.01$. PCoA plots of microbiota beta diversity based on the Bray-Curtis dissimilarity metric comparing ME/CFS and healthy controls (D) and groups stratified by sr-IBS status (E). Differences based on PERMANOVA (unadjusted) (D, E) or PERMANOVA-FL (adjusted for covariates) (D) are shown below for each group comparison with associated pseudo-f statistic and p-value (D) or the Bonferroni adjusted p-value ( $\left.p^{\text {adj }}\right)(\mathbf{E})$. 
A

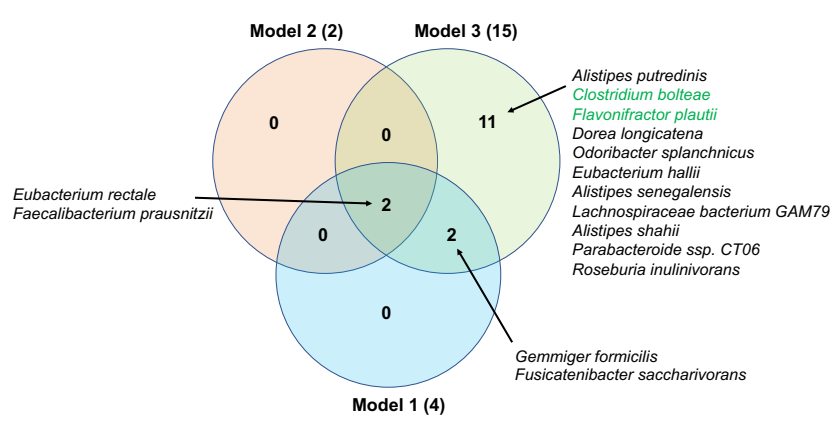

B
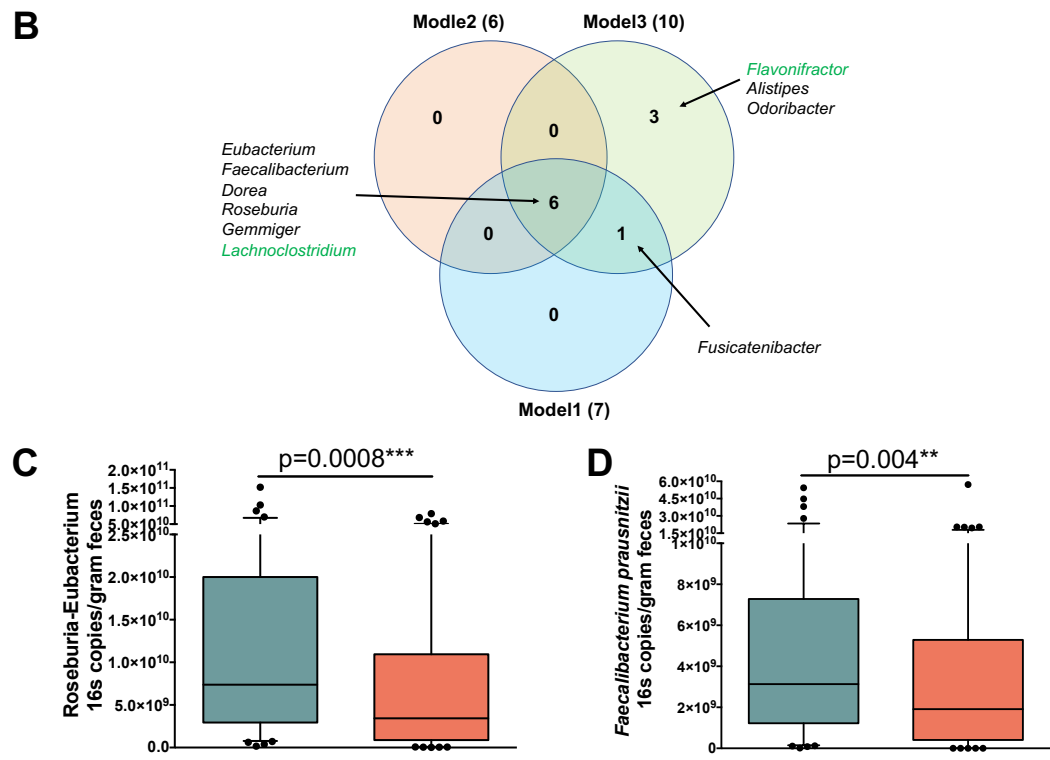

$\mathbf{E}$

$\mathrm{p}^{\mathrm{adj}}=0.011^{*}$

$\mathbf{F}$

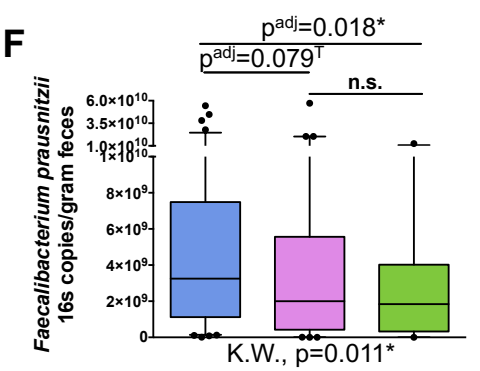

G

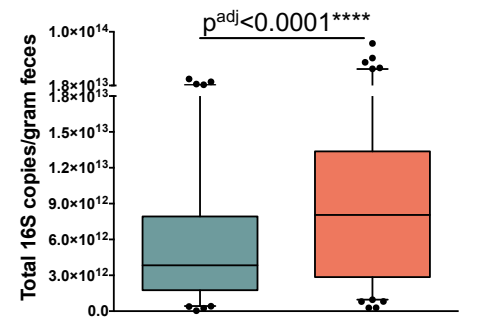

H

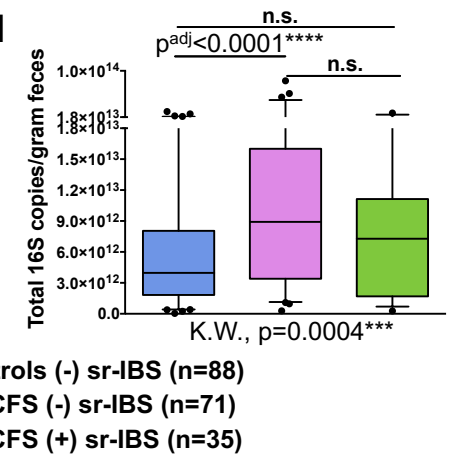

1049 Figure 2: Differential abundance and quantitation of fecal bacteria. Venn diagram showing the results of multivariate GAMLSS-BEZI models for differentially abundant 1051 species (A) and genera (B) from four models: Model 1: comparing All ME/CFS vs healthy 
1052 controls, Model 2: comparing ME/CFS without sr-IBS vs healthy controls without sr-IBS, 1053 Model 3: comparing ME/CFS with sr-IBS vs healthy controls without sr-IBS, and Model 4: 1054 comparing ME/CFS without sr-IBS vs ME/CFS with sr-IBS. Models are adjusted for covariates: sr-IBS diagnosis (only model 1), site of recruitment, sex, BMI, race and ethnicity, age, antibiotic use within 6-12 weeks of sample collection, probiotic supplement use, and prebiotic supplement use. Note: no taxa reached significance in Model 4 after FDR adjustment, thus only Models 1-3 are shown. Also see Supplementary Tables 1A, B. Box-and-whiskers plots showing the distribution of Roseburia-Eubacterium 16S rRNA and $F$. prausnitzii $16 \mathrm{~S}$ rRNA per gram of feces determined by qPCR between ME/CFS and healthy controls (C, D) and among stratified groups for healthy controls without (-) srIBS, ME/CFS subjects without (-) sr-IBS, and ME/CFS subjects with (+) sr-IBS (E, F). Box-and-whiskers plots showing the distribution of Total bacterial 16S rRNA genes per gram of feces determined by qPCR between ME/CFS and healthy controls (G) and among stratified groups $(\mathbf{H})$. Statistical significance was determined based on two-tailed $p$-values from the Mann-Whitney $U$ test (C, D, G). For stratified analyses significance was first determined based on the Kruskal-Wallis test (K.W., results shown below each figure in $\mathbf{E}, \mathbf{F}, \mathbf{H})$. If significant $(p<0.05)$ based on K.W., then between group significance was determined based on the Mann-Whitney $U$ test with multiple testing (Bonferroni)

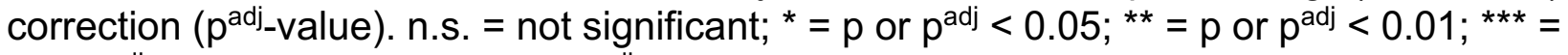
$p$ or $p^{\text {adj }}<0.001 ; T=$ trend $\left(p\right.$ or $\left.p^{\text {adj }}<0.1\right)$. 

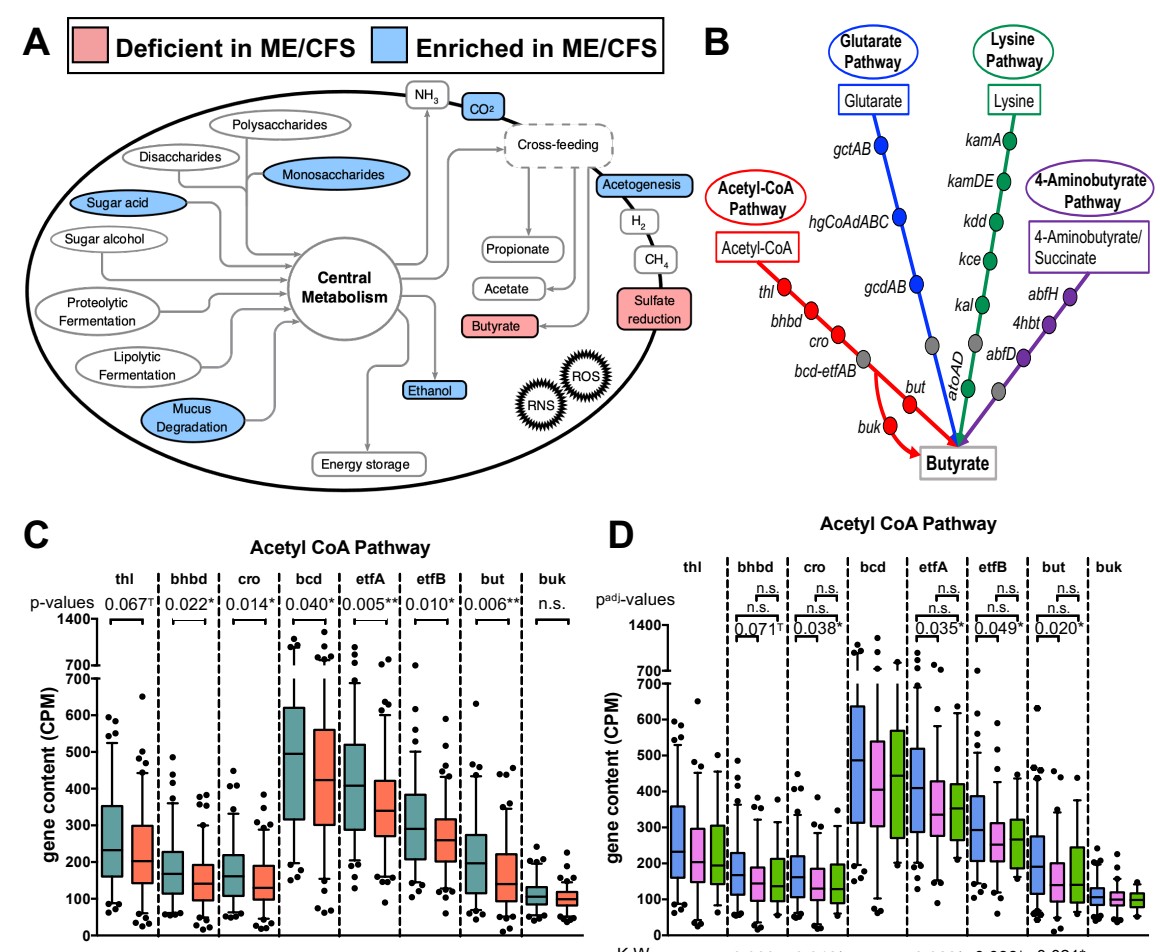

E

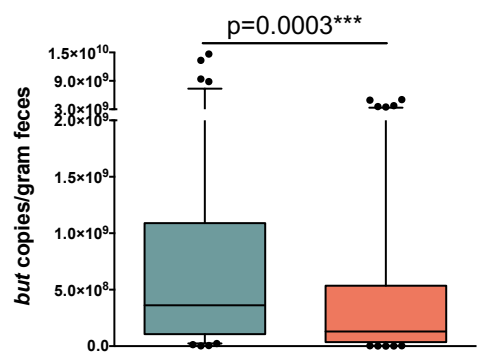

$\mathbf{F}$
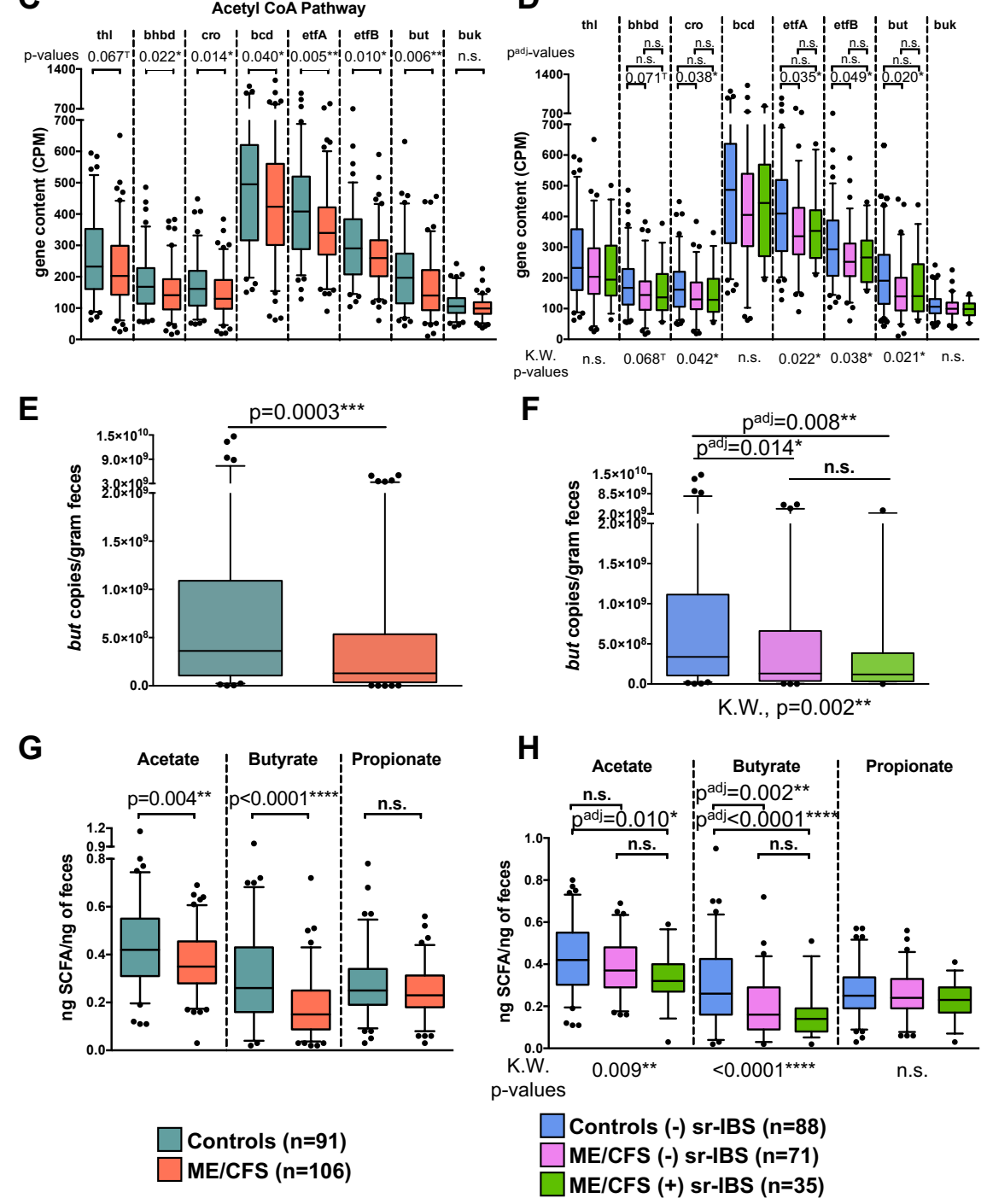

1073 Figure 3: Functional metagenomic and metabolomic analyses. Gut bacterial metabolic 1074 processes that differ (deficient or enriched) in ME/CFS compared with healthy controls 1075 (A). Schematic showing the 4 gut bacterial pathways of butyrate production and the 
1076 bacterial genes involved in the production of butyrate (B). Note: gray circles indicate that 1077 the bcd-etfAB complex is shared among all 4 pathways. Box-and-whiskers plots showing 1078 the distribution of gene counts (CPM) for genes in the acetyl-CoA pathway of butyrate 1079 production (C, D) between ME/CFS and healthy controls (C) and among stratified groups 1080 (D). Box-and-whiskers plots showing the distribution of fecal but gene copies per gram of 1081 feces determined by GPCR between ME/CFS and healthy controls (E) and among 1082 stratified groups (F). Box-and-whiskers plots showing the distribution of fecal SCFAs 1083 measured by GC-MS between ME/CFS and healthy controls (G) and among stratified 1084 groups $(\mathbf{H})$. Statistical significance was determined based on two-tailed p-values from the 1085 Mann-Whitney U test for each gene (C, E, G). For stratified analyses significance was 1086 first determined based on the Kruskal-Wallis test for each gene (K.W., results shown 1087 below each figure in $\mathbf{D}, \mathbf{F}, \mathbf{H})$. If significant $(p<0.05)$ based on K.W., then between group 1088 significance was determined based on the Mann-Whitney $U$ test with multiple testing 1089 (Bonferroni) correction ( $p^{\text {adj-value) }}$. n.s. $=$ not significant; ${ }^{*}=p$ or $p^{\text {adj }}<0.05 ;{ }^{* *}=p$ or $p^{\text {adj }}$ 1090 $<0.01 ;{ }^{* * *}=p$ or $p^{\text {adj }}<0.001 ;{ }^{* * *}=p$ or $p^{\text {adj }}<0.0001 ; T=$ trend $\left(p\right.$ or $\left.p^{\text {adj }}<0.1\right)$. 


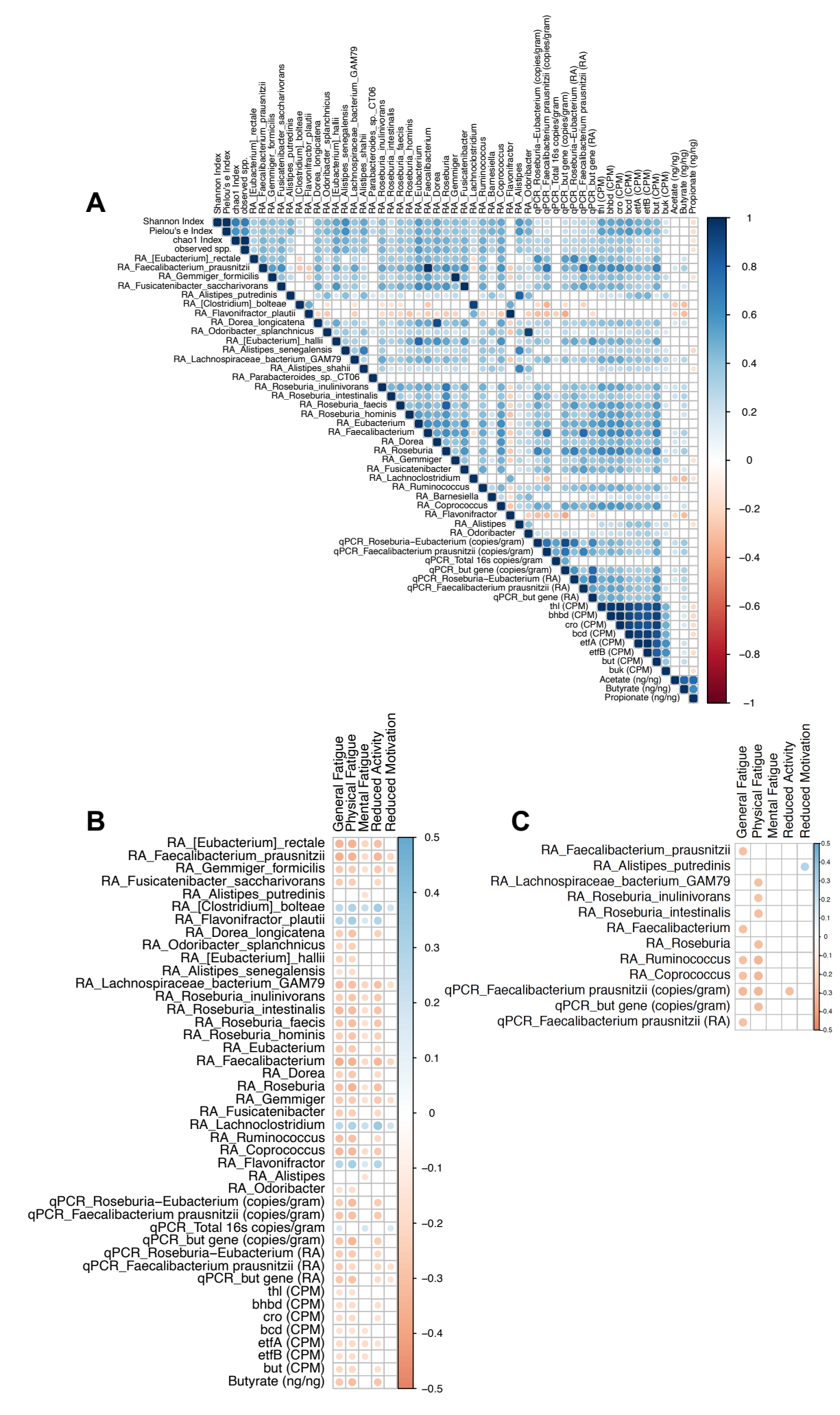

1093 Figure 4: Spearman's correlation analysis among measured variables and fatigue 1094 scores. Spearman's correlation assessing relationships among all measured variables in 1095 both cases and controls $(n=197)(\mathbf{A})$, and between measured variables and fatigue 
1096 dimension scores in both cases and controls $(n=197)(B)$, and only within ME/CFS cases 1097 ( $n=106)(C)$. Note: no significant correlations between measured variables and fatigue 1098 scores were observed within healthy controls $(n=91)$, only within ME/CFS cases without 1099 sr-IBS $(n=71)$, or only within ME/CFS cases with sr-IBS $(n=35)$. Each colored circle in 1100 each correlogram represents a significant correlation (nonsignificant correlations are 1101 white). The color bars show the scale for the Spearman's rank correlation coefficient. Both 1102 the size and color of the circles indicate the strength of the correlation. FDR was controlled 1103 with the Benjamini-Hochberg procedure, with correlations with adjusted $p$-values $<0.05$ 1104 shown. In figures $\mathbf{B}$ and $\mathbf{C}$, all measured variables (shown in A) were compared against 1105 all five fatigue dimensions, and only significant correlations are shown (all other 1106 comparisons were not significant after adjustment). 


\section{$\underline{\text { Tables }}$}

Table 1: Cohort characteristics

\begin{tabular}{|c|c|c|c|}
\hline & ME/CFS $(n=106)$ & Control $(n=91)$ & P-value \\
\hline Age (Mean \pm SD) & $47.8 \pm 13.7$ & $47.0 \pm 14.1$ & $0.78^{\mathrm{a}}$ \\
\hline BMI (Mean \pm SD) & $26.1 \pm 5.2$ & $25.2 \pm 4.7$ & $0.31^{\mathrm{a}}$ \\
\hline Sex (Male|Female) & $31 \mid 75$ & $22 \mid 69$ & $0.52^{\mathrm{b}}$ \\
\hline sr-IBS (Yes|No) & $35 \mid 71$ & $3 \mid 88$ & $<0.001^{b}$ \\
\hline Antibiotic use, 6-12 weeks (Yes|No) & $13 \mid 93$ & $5 \mid 86$ & $0.14^{\mathrm{b}}$ \\
\hline Prebiotic supplement use (Yes|No) & $11 \mid 95$ & $2 \mid 89$ & $\mathbf{0 . 0 2}{ }^{\mathrm{b}}$ \\
\hline Probiotic supplement use (Yes|No) & $41 \mid 65$ & $10 \mid 81$ & $<0.001^{b}$ \\
\hline Duration of ME/CFS > 3 years (Yes|No) & $98 \mid 8$ & $\mathrm{~N} / \mathrm{A}$ & $\mathrm{N} / \mathrm{A}$ \\
\hline \multicolumn{4}{|l|}{ Site } \\
\hline Palo Alto, $C A$ & $19(18 \%)$ & $18(19 \%)$ & \multirow{5}{*}{$0.30^{\mathrm{c}}$} \\
\hline Salt Lake City, UT & $32(30 \%)$ & $32(35 \%)$ & \\
\hline Incline Village, $\mathrm{NV}$ & $23(22 \%)$ & $17(19 \%)$ & \\
\hline New York, $N Y$ & $17(16 \%)$ & $19(21 \%)$ & \\
\hline Miami, FL & $15(14 \%)$ & $5(6 \%)$ & \\
\hline \multicolumn{4}{|l|}{ Race } \\
\hline Hispanic & $6(6 \%)$ & $3(3 \%)$ & \multirow{3}{*}{$0.40^{\mathrm{c}}$} \\
\hline White \& Non-Hispanic & $93(88 \%)$ & $85(94 \%)$ & \\
\hline Non-White \& Non-Hispanic & $7(6 \%)$ & $3(3 \%)$ & \\
\hline
\end{tabular}

a P-values based on Mann-Whitney $U$ test

${ }^{b} P$-values based on Fisher's Exact Test

c $P$-values based on chi-squared test 
Table 2: Generalized linear regression showing the relationship between ME/CFS status and alpha diversity metrics.

\begin{tabular}{|c|c|c|c|c|c|c|c|c|c|}
\hline \multirow[b]{2}{*}{ Predictors } & \multicolumn{3}{|c|}{ shannon } & \multicolumn{3}{|c|}{ evenness } & \multicolumn{3}{|c|}{ observed species } \\
\hline & $\beta_{E s t}$ & $95 \% C I$ & $p$ & $\beta_{E s t}$ & $95 \% C I$ & $p$ & Fold Change & $95 \% C I$ & $p$ \\
\hline (Intercept) & 0.63 & $0.60-0.67$ & $<0.001$ & -0.31 & $-0.34--0.28$ & $<0.001$ & 432.26 & $400.00-467.11$ & $<0.001$ \\
\hline $\mathrm{ME} / \mathrm{CFS}$ & -0.02 & $-0.05-0.00$ & 0.103 & -0.02 & $-0.04-0.00$ & 0.096 & 0.98 & $0.93-1.03$ & 0.481 \\
\hline sr-IBS & -0.03 & $-0.06-0.00$ & 0.054 & -0.03 & $-0.06-0.00$ & 0.032 & 1.01 & $0.94-1.07$ & 0.865 \\
\hline Site 2(Florida) & 0.00 & $-0.05-0.05$ & 0.973 & 0.00 & $-0.04-0.04$ & 0.954 & 0.99 & $0.90-1.09$ & 0.859 \\
\hline Site 3(Nevada) & -0.02 & $-0.06-0.02$ & 0.246 & -0.02 & $-0.05-0.02$ & 0.279 & 0.95 & $0.88-1.03$ & 0.222 \\
\hline Site 4(New York) & 0.01 & $-0.03-0.04$ & 0.673 & 0.00 & $-0.03-0.04$ & 0.814 & 1.05 & $0.98-1.14$ & 0.166 \\
\hline Site $5($ Utah) & 0.00 & $-0.03-0.04$ & 0.863 & 0.00 & $-0.03-0.03$ & 0.957 & 1.02 & $0.95-1.10$ & 0.522 \\
\hline Sex & -0.01 & $-0.04-0.02$ & 0.582 & -0.01 & $-0.03-0.02$ & 0.669 & 0.97 & $0.91-1.03$ & 0.268 \\
\hline BMI & 0.00 & $-0.01-0.01$ & 0.920 & 0.00 & $-0.01-0.01$ & 0.726 & 0.98 & $0.96-1.00$ & 0.119 \\
\hline Hispanic & 0.01 & $-0.05-0.06$ & 0.850 & 0.00 & $-0.05-0.06$ & 0.886 & 1.02 & $0.90-1.15$ & 0.758 \\
\hline Non-White \& Non-Hispanic & -0.02 & $-0.07-0.03$ & 0.370 & -0.02 & $-0.07-0.03$ & 0.395 & 0.94 & $0.85-1.05$ & 0.291 \\
\hline Age & 0.02 & $0.00-0.03$ & 0.016 & 0.01 & $0.00-0.02$ & 0.023 & 1.03 & $1.01-1.06$ & 0.011 \\
\hline Antibiotics & 0.01 & $-0.03-0.05$ & 0.677 & 0.01 & $-0.02-0.05$ & 0.520 & 0.96 & $0.88-1.04$ & 0.292 \\
\hline Probiotics & 0.02 & $-0.01-0.05$ & 0.237 & 0.02 & $-0.01-0.04$ & 0.186 & 1.00 & $0.94-1.06$ & 0.871 \\
\hline Prebiotics & -0.04 & $-0.09-0.01$ & 0.125 & -0.03 & $-0.08-0.01$ & 0.133 & 0.94 & $0.85-1.04$ & 0.223 \\
\hline
\end{tabular}

Results for each outcome variable were estimated using linear regression (Shannon and evenness) or a negative binomial generalized linear model (observed species) and adjusted for covariates.

$\beta_{\text {Est }}$ (Estimate) or Fold Change, 95\% Confidence Intervals (Cl), and p-values are shown for each model.

Significant predictors $(p<0.05)$ are indicated in bold. 
Table 3: Generalized linear regression showing the relationship between ME/CFS status and fecal quantities of bacterial taxa and the but gene by qPCR.

\begin{tabular}{|c|c|c|c|c|c|c|c|c|c|c|c|c|}
\hline \multirow[b]{2}{*}{ Predictors } & \multicolumn{3}{|c|}{ Roseburia/Eubacterium 16S } & \multicolumn{3}{|c|}{ F. prausnitzii 16S } & \multicolumn{3}{|c|}{ total $16 \mathrm{~S}$} & \multicolumn{3}{|c|}{ but gene } \\
\hline & Fold Change & e $\quad 95 \% \mathrm{CI}$ & $p$ & Fold Chang & ge $95 \% \mathrm{CI}$ & $p$ & Fold Change & $95 \% C I$ & $p$ & Fold Chang & ge $95 \% C I$ & $p$ \\
\hline (Intercept) & $1 \mathrm{E}+10$ & $8 \mathrm{E}+9-3 \mathrm{E}+10$ & $<0.001$ & $7.03 \mathrm{E}+9$ & $3 \mathrm{E}+9-1 \mathrm{E}+10$ & $<0.001$ & $4 \mathrm{E}+12$ & $3 \mathrm{E}+12-7 \mathrm{E}+12$ & $<0.001$ & $8 \mathrm{E}+8$ & $3 \mathrm{E}+8-2 \mathrm{E}+09$ & $<0.001$ \\
\hline $\mathrm{ME} / \mathrm{CFS}$ & 0.56 & $0.33-0.93$ & 0.026 & 0.61 & $0.38-0.98$ & 0.043 & 2.08 & $1.52-2.83$ & $<0.001$ & 0.51 & $0.28-0.93$ & 0.028 \\
\hline sr-IBS & 0.69 & $0.38-1.28$ & 0.245 & 0.74 & $0.42-1.31$ & 0.301 & 0.76 & $0.52-1.09$ & 0.139 & 0.63 & $0.30-1.28$ & 0.203 \\
\hline Site 2 (Florida) & 1.99 & $0.80-4.97$ & 0.141 & 1.63 & $0.70-3.82$ & 0.262 & 2.09 & $1.21-3.62$ & 0.009 & 1.79 & $0.61-5.22$ & 0.288 \\
\hline Site 3 (Nevada) & 1.41 & $0.65-3.06$ & 0.382 & 1.14 & $0.56-2.35$ & 0.715 & 1.38 & $0.87-2.20$ & 0.172 & 1.82 & $0.74-4.50$ & 0.195 \\
\hline Site 4 (New York) & 1.24 & $0.60-2.57$ & 0.554 & 1.21 & $0.61-2.37$ & 0.586 & 0.86 & $0.56-1.33$ & 0.510 & 0.95 & $0.41-2.23$ & 0.915 \\
\hline Site 5 (Utah) & 1.12 & $0.57-2.20$ & 0.742 & 1.09 & $0.58-2.04$ & 0.792 & 1.20 & $0.80-1.80$ & 0.375 & 1.22 & $0.55-2.69$ & 0.627 \\
\hline Sex & 0.81 & $0.46-1.43$ & 0.463 & 0.70 & $0.41-1.20$ & 0.194 & 0.94 & $0.67-1.32$ & 0.723 & 1.04 & $0.53-2.03$ & 0.910 \\
\hline BMI & 0.79 & $0.63-1.00$ & 0.052 & 0.80 & $0.64-0.99$ & 0.043 & 0.81 & $0.71-0.94$ & 0.004 & 0.82 & $0.62-1.07$ & 0.148 \\
\hline Hispanic & 0.48 & $0.16-1.46$ & 0.195 & 0.86 & $0.30-2.43$ & 0.776 & 1.12 & $0.57-2.19$ & 0.745 & 0.30 & $0.08-1.13$ & 0.076 \\
\hline Non-White \& Non-Hispanic & 1.80 & $0.66-4.91$ & 0.256 & 1.91 & $0.75-4.87$ & 0.178 & 1.42 & $0.78-2.59$ & 0.258 & 2.72 & $0.84-8.84$ & 0.098 \\
\hline Age & 0.99 & $0.78-1.27$ & 0.951 & 0.98 & $0.78-1.23$ & 0.864 & 1.10 & $0.95-1.27$ & 0.200 & 1.17 & $0.88-1.55$ & 0.289 \\
\hline Antibiotics & 0.70 & $0.32-1.55$ & 0.379 & 0.44 & $0.21-0.91$ & 0.029 & 0.50 & $0.31-0.81$ & 0.006 & 0.35 & $0.14-0.90$ & 0.030 \\
\hline Probiotics & 1.03 & $0.59-1.80$ & 0.912 & 1.42 & $0.84-2.38$ & 0.189 & 1.11 & $0.79-1.55$ & 0.553 & 1.00 & $0.52-1.92$ & 0.993 \\
\hline Prebiotics & 0.67 & $0.25-1.74$ & 0.408 & 0.73 & $0.30-1.79$ & 0.494 & 0.72 & $0.40-1.28$ & 0.259 & 0.61 & $0.20-1.88$ & 0.391 \\
\hline
\end{tabular}

Results for each outcome variable were estimated using generalized linear regression with a Gamma distribution with log link and adjusted for covariates.

Fold Change, 95\% Confidence Intervals $(\mathrm{Cl})$, and p-values $(p)$ are shown for each model.

Significant predictors $(p<0.05)$ are indicated in bold. 
Table 4: Generalized linear regression showing the relationship between ME/CFS status and metagenomic gene content in the acetyl-CoA pathway.

\begin{tabular}{|c|c|c|c|c|c|c|c|c|c|c|c|c|}
\hline \multirow[b]{3}{*}{ Predictors } & \multicolumn{12}{|c|}{ Acetyl-CoA pathway } \\
\hline & \multicolumn{3}{|c|}{ thl } & \multicolumn{3}{|c|}{ bhbd } & \multicolumn{3}{|c|}{ cro } & \multicolumn{3}{|c|}{ bed } \\
\hline & Fold Change & $95 \% C I$ & $p$ & Fold Change & $95 \% C I$ & $p$ & Fold Change & $95 \% C I$ & $p$ & Fold Change & $95 \% C I$ & $p$ \\
\hline (Intercept) & 275.78 & $216-353$ & $<0.001$ & 203.9 & $160-260$ & $<0.001$ & 192.18 & $152-244$ & $<0.001$ & 523.23 & $420-655$ & $<0.001$ \\
\hline $\mathrm{ME} / \mathrm{CFS}$ & 0.86 & $0.72-1.03$ & 0.087 & 0.81 & $0.68-0.96$ & 0.014 & 0.80 & $0.68-0.95$ & 0.008 & 0.85 & $0.73-1.00$ & 0.043 \\
\hline sr-IBS & 1.02 & $0.83-1.25$ & 0.874 & 1.08 & $0.88-1.32$ & 0.465 & 1.07 & $0.88-1.31$ & 0.473 & 1.06 & $0.88-1.28$ & 0.522 \\
\hline Site 2 (Florida) & 1.04 & $0.77-1.41$ & 0.806 & 1.03 & $0.76-1.39$ & 0.868 & 1.01 & $0.75-1.35$ & 0.970 & 0.97 & $0.74-1.28$ & 0.839 \\
\hline Site 3 (Nevada) & 1.06 & $0.83-1.36$ & 0.634 & 0.99 & $0.78-1.27$ & 0.964 & 1.01 & $0.79-1.28$ & 0.951 & 1.00 & $0.80-1.25$ & 0.987 \\
\hline Site 4 (New York) & 1.01 & $0.79-1.28$ & 0.962 & 0.97 & $0.76-1.23$ & 0.795 & 0.96 & $0.76-1.22$ & 0.753 & 0.98 & $0.78-1.21$ & 0.819 \\
\hline Site 5 (Utah) & 1.02 & $0.81-1.27$ & 0.877 & 0.98 & $0.78-1.21$ & 0.828 & 0.98 & $0.79-1.21$ & 0.837 & 0.99 & $0.81-1.21$ & 0.915 \\
\hline Sex & 0.92 & $0.76-1.10$ & 0.356 & 0.87 & $0.72-1.05$ & 0.149 & 0.89 & $0.75-1.07$ & 0.227 & 0.96 & $0.81-1.14$ & 0.670 \\
\hline BMI & 0.94 & $0.87-1.02$ & 0.110 & 0.94 & $0.87-1.01$ & 0.103 & 0.96 & $0.89-1.03$ & 0.221 & 0.97 & $0.91-1.04$ & 0.426 \\
\hline Hispanic & 0.98 & $0.68-1.46$ & 0.929 & 0.95 & $0.66-1.41$ & 0.802 & 0.96 & $0.67-1.41$ & 0.821 & 1.12 & $0.80-1.61$ & 0.499 \\
\hline Non-White \& Non-Hispanic & 0.96 & $0.69-1.36$ & 0.796 & 1.00 & $0.73-1.41$ & 0.993 & 0.97 & $0.71-1.37$ & 0.874 & 0.95 & $0.71-1.30$ & 0.736 \\
\hline Age & 1.01 & $0.93-1.09$ & 0.790 & 1.00 & $0.93-1.09$ & 0.926 & 1.00 & $0.93-1.08$ & 0.907 & 1.01 & $0.94-1.09$ & 0.695 \\
\hline Antibiotics & 0.90 & $0.69-1.18$ & 0.410 & 0.95 & $0.74-1.25$ & 0.703 & 0.92 & $0.71-1.19$ & 0.499 & 0.86 & $0.68-1.11$ & 0.224 \\
\hline Probiotics & 0.99 & $0.82-1.19$ & 0.876 & 1.00 & $0.83-1.21$ & 0.998 & 1.00 & $0.84-1.20$ & 0.957 & 1.03 & $0.87-1.22$ & 0.709 \\
\hline Prebiotics & 1.08 & $0.80-1.50$ & 0.622 & 1.07 & $0.79-1.48$ & 0.654 & 1.06 & $0.79-1.45$ & 0.712 & 1.04 & $0.79-1.40$ & 0.795 \\
\hline
\end{tabular}

(Part-2/2)

\begin{tabular}{|c|c|c|c|c|c|c|c|c|c|c|c|c|}
\hline \multirow[b]{3}{*}{ Predictors } & \multicolumn{12}{|c|}{ Acetyl-CoA pathway } \\
\hline & \multicolumn{3}{|c|}{ etfA } & \multicolumn{3}{|c|}{ etfB } & \multicolumn{3}{|c|}{ but } & \multicolumn{3}{|c|}{ buk } \\
\hline & Fold Change & $95 \% C I$ & $p$ & Fold Change & $95 \% C I$ & $p$ & Fold Change & $95 \% C I$ & $p$ & Fold Change & $95 \% C I$ & $p$ \\
\hline (Intercept) & 455.1 & $383-542$ & $<0.001$ & 330.2 & $278-392$ & $<0.001$ & 237.18 & $182-311$ & $<0.001$ & 114.07 & $98-132$ & $<0.001$ \\
\hline $\mathrm{ME} / \mathrm{CFS}$ & 0.84 & $0.74-0.95$ & 0.005 & 0.85 & $0.75-0.96$ & 0.007 & 0.75 & $0.62-0.91$ & 0.003 & 0.93 & $0.84-1.04$ & 0.180 \\
\hline sr-IBS & 1.03 & $0.89-1.19$ & 0.731 & 1.03 & $0.89-1.19$ & 0.675 & 1.08 & $0.87-1.36$ & 0.493 & 0.94 & $0.83-1.07$ & 0.345 \\
\hline Site 2 (Florida) & 0.97 & $0.78-1.20$ & 0.759 & 0.96 & $0.78-1.19$ & 0.713 & 0.97 & $0.70-1.36$ & 0.869 & 0.91 & $0.76-1.10$ & 0.339 \\
\hline Site 3 (Nevada) & 0.99 & $0.83-1.18$ & 0.879 & 0.99 & $0.83-1.18$ & 0.903 & 0.95 & $0.72-1.25$ & 0.720 & 1.08 & $0.93-1.26$ & 0.339 \\
\hline Site 4 (New York) & 0.91 & $0.77-1.08$ & 0.274 & 0.92 & $0.77-1.09$ & 0.311 & 0.94 & $0.72-1.22$ & 0.644 & 0.87 & $0.75-1.01$ & 0.061 \\
\hline Site 5 (Utah) & 0.97 & $0.83-1.14$ & 0.713 & 0.98 & $0.84-1.14$ & 0.776 & 0.96 & $0.75-1.23$ & 0.773 & 0.98 & $0.86-1.12$ & 0.782 \\
\hline Sex & 0.92 & $0.81-1.05$ & 0.244 & 0.94 & $0.82-1.07$ & 0.332 & 0.86 & $0.70-1.06$ & 0.165 & 0.99 & $0.88-1.10$ & 0.804 \\
\hline BMI & 0.99 & $0.94-1.05$ & 0.810 & 1.00 & $0.95-1.05$ & 0.976 & 0.92 & $0.84-1.00$ & 0.045 & 1.02 & $0.97-1.07$ & 0.389 \\
\hline Hispanic & 1.08 & $0.83-1.42$ & 0.556 & 1.09 & $0.84-1.43$ & 0.503 & 0.89 & $0.60-1.37$ & 0.563 & 1.05 & $0.84-1.33$ & 0.660 \\
\hline Non-White \& Non-Hispanic & 0.91 & $0.72-1.16$ & 0.411 & 0.91 & $0.73-1.16$ & 0.430 & 1.09 & $0.77-1.60$ & 0.637 & 0.92 & $0.76-1.14$ & 0.437 \\
\hline Age & 1.02 & $0.96-1.08$ & 0.582 & 1.02 & $0.96-1.08$ & 0.535 & 0.99 & $0.90-1.08$ & 0.760 & 1.02 & $0.97-1.07$ & 0.414 \\
\hline Antibiotics & 0.94 & $0.78-1.14$ & 0.528 & 0.93 & $0.78-1.13$ & 0.453 & 0.99 & $0.75-1.34$ & 0.966 & 0.97 & $0.83-1.15$ & 0.754 \\
\hline Probiotics & 1.02 & $0.89-1.16$ & 0.790 & 1.03 & $0.90-1.17$ & 0.669 & 1.03 & $0.84-1.26$ & 0.763 & 0.98 & $0.87-1.09$ & 0.665 \\
\hline Prebiotics & 1.07 & $0.86-1.34$ & 0.577 & 1.07 & $0.87-1.34$ & 0.532 & 0.94 & $0.68-1.34$ & 0.733 & 1.13 & $0.93-1.38$ & 0.216 \\
\hline
\end{tabular}

Results for each outcome variable were estimated using generalized linear regression with a Gamma distribution with log link and adjusted for covariates.

Fold Change, 95\% Confidence Intervals $(\mathrm{Cl})$, and $p$-values $(p)$ are shown for each model.

Significant predictors $(p<0.05)$ are indicated in bold. 
Table 5: Generalized linear regression showing the relationship between ME/CFS status and SCFAs.

\begin{tabular}{|c|c|c|c|c|c|c|c|c|c|}
\hline \multirow[b]{2}{*}{ Predictors } & \multicolumn{3}{|c|}{ Acetate } & \multicolumn{3}{|c|}{ Butyrate } & \multicolumn{3}{|c|}{ Propionate } \\
\hline & Fold Change & $C I$ & $p$ & Fold Change & CI & $p$ & Fold Change & $C I$ & $p$ \\
\hline (Intercept) & 0.46 & $0.39-0.54$ & $<0.001$ & 0.36 & $0.26-0.48$ & $<0.001$ & 0.31 & $0.25-0.38$ & $<0.001$ \\
\hline $\mathrm{ME} / \mathrm{CFS}$ & 0.87 & $0.77-0.98$ & 0.019 & 0.61 & $0.49-0.75$ & $<0.001$ & 0.90 & $0.79-1.04$ & 0.164 \\
\hline sr-IBS & 1.92 & $1.28-2.87$ & 0.002 & 2.11 & $1.00-4.44$ & 0.052 & 2.20 & $1.35-3.58$ & 0.002 \\
\hline $\mathrm{ME} / \mathrm{CFS} * \mathrm{sr}-\mathrm{IBS}$ & 0.48 & $0.31-0.73$ & $<0.001$ & 0.41 & $0.19-0.91$ & 0.031 & 0.40 & $0.24-0.67$ & $<0.001$ \\
\hline Site 2 (Florida) & 1.25 & $1.02-1.53$ & 0.035 & 1.23 & $0.85-1.80$ & 0.274 & 1.17 & $0.91-1.49$ & 0.222 \\
\hline Site 3 (Nevada) & 1.02 & $0.86-1.21$ & 0.811 & 1.10 & $0.80-1.50$ & 0.560 & 0.98 & $0.80-1.20$ & 0.838 \\
\hline Site 4 (New York) & 0.92 & $0.78-1.07$ & 0.282 & 0.83 & $0.62-1.11$ & 0.210 & 0.88 & $0.73-1.07$ & 0.200 \\
\hline Site 5 (Utah) & 0.93 & $0.80-1.08$ & 0.370 & 1.03 & $0.78-1.36$ & 0.819 & 0.93 & $0.77-1.11$ & 0.419 \\
\hline Sex & 0.93 & $0.82-1.05$ & 0.264 & 0.73 & $0.58-0.92$ & 0.009 & 0.88 & $0.76-1.03$ & 0.105 \\
\hline BMI & 1.04 & $0.99-1.09$ & 0.149 & 1.00 & $0.91-1.10$ & 0.931 & 1.08 & $1.01-1.15$ & 0.016 \\
\hline Hispanic & 1.01 & $0.78-1.30$ & 0.942 & 1.44 & $0.90-2.31$ & 0.132 & 0.96 & $0.70-1.30$ & 0.777 \\
\hline Non-White \& Non-Hispanic & 1.06 & $0.85-1.33$ & 0.579 & 1.21 & $0.80-1.84$ & 0.356 & 1.21 & $0.93-1.59$ & 0.163 \\
\hline Age & 0.94 & $0.89-0.99$ & 0.026 & 0.92 & $0.83-1.01$ & 0.096 & 0.96 & $0.90-1.02$ & 0.181 \\
\hline Antibiotics & 0.87 & $0.73-1.03$ & 0.107 & 0.89 & $0.65-1.23$ & 0.493 & 1.00 & $0.82-1.24$ & 0.963 \\
\hline Probiotics & 0.97 & $0.86-1.10$ & 0.640 & 1.03 & $0.82-1.30$ & 0.767 & 0.99 & $0.85-1.15$ & 0.899 \\
\hline Prebiotics & 1.01 & $0.82-1.25$ & 0.906 & 1.05 & $0.71-1.55$ & 0.822 & 1.08 & $0.83-1.39$ & 0.567 \\
\hline Model 2: ME/CFS(-)sr-IBS & 0.87 & $0.77-0.98$ & 0.022 & 0.60 & $0.48-0.75$ & $<0.001$ & 0.90 & $0.78-1.04$ & 0.149 \\
\hline Model 3: $\mathrm{ME} / \mathrm{CFS}(+)$ sr-IBS & 0.83 & $0.71-0.96$ & 0.017 & 0.56 & $0.42-0.73$ & $<0.001$ & 0.83 & $0.70-1.00$ & 0.047 \\
\hline Model 4: sr-IBS & 0.91 & $0.79-1.05$ & 0.186 & 0.88 & $0.66-1.17$ & 0.379 & 0.88 & $0.73-1.05$ & 0.154 \\
\hline
\end{tabular}

Results for each outcome variable were estimated using generalized linear regression with a Gamma distribution with log link and adjusted for covariates.

Fold Change, 95\% Confidence Intervals $(\mathrm{Cl})$, and p-values $(p)$ are shown for each model.

Note: The interaction term ME/CFS*sr-IBS was significant in all three models, therefore the association of each SCFA with ME/CFS and sr-IBS status was evaluated in stratified models (Models 2-4) using generalized linear regression with a Gamma distribution and log link and adjusted for covariates (bottom three rows of the table): Model 2= ME/CFS(-)sr-IBS vs Controls(-)sr-IBS; Model 3= ME/CFS(+)sr-IBS vs Controls(-)sr-IBS; Model 4= ME/CFS(+)sr-IBS vs ME/CFS(-)sr-IBS. Complete results for Models 2-4 can be found in Supplementary Tables 5A-C.

Significant predictors $(p<0.05)$ are indicated in bold. 


\section{References}

1. Fukuda, K., et al., The chronic fatigue syndrome: a comprehensive approach to its definition and study. International Chronic Fatigue Syndrome Study Group. Ann Intern Med, 1994. 121(12): p. 953-9.

2. Carruthers, B.M., et al., Myalgic encephalomyelitis: International Consensus Criteria. J Intern Med, 2011. 270(4): p. 327-38.

3. Institute of Medicine, in Beyond Myalgic Encephalomyelitis/Chronic Fatigue Syndrome: Redefining an Illness, The National Academies Collection: Reports funded by National Institutes of Health. 2015: Washington (DC).

4. Slomko, J., et al., Prevalence and characteristics of chronic fatigue syndrome/myalgic encephalomyelitis (CFS/ME) in Poland: a cross-sectional study. BMJ Open, 2019. 9(3): p. e023955.

5. Johnston, S., et al., The prevalence of chronic fatigue syndromel myalgic encephalomyelitis: a meta-analysis. Clin Epidemiol, 2013. 5: p. 105-10.

6. Lorusso, L., et al., Immunological aspects of chronic fatigue syndrome. Autoimmun Rev, 2009. 8(4): p. 287-91.

7. Lim, E.J., et al., Systematic review and meta-analysis of the prevalence of chronic fatigue syndrome/myalgic encephalomyelitis (CFS/ME). J Transl Med, 2020. 18(1): p. 100.

8. Jason, L.A., et al., The economic impact of ME/CFS: individual and societal costs. Dyn Med, 2008. 7: p. 6.

9. $\quad$ Carruthers, B.M., et al., Myalgic Encephalomyelitis/Chronic Fatigue Syndrome: Clinical Working Case Definition, Diagnostic and Treatment Protocols. Chronic Fatigue Syndrome, 2003. 11(1): p. 7-115. 
10. Clayton, E., Beyond Myalgic Encephalomyelitis/Chronic Fatigue Syndrome: redefining an illness. Washington (DC): The National Academies Collection, 2015.

11. Komaroff, A.L. and W.I. Lipkin, Insights from myalgic encephalomyelitis/chronic fatigue syndrome may help unravel the pathogenesis of postacute COVID-19 syndrome. Trends Mol Med, 2021. 27(9): p. 895-906.

12. Paul, B.D., et al., Redox imbalance links COVID-19 and myalgic encephalomyelitis/chronic fatigue syndrome. Proc Natl Acad Sci U S A, 2021. 118(34).

13. Hickie, I., et al., Post-infective and chronic fatigue syndromes precipitated by viral and non-viral pathogens: prospective cohort study. BMJ, 2006. 333(7568): p. 575.

14. Underhill, R.A., Myalgic encephalomyelitis, chronic fatigue syndrome: An infectious disease. Med Hypotheses, 2015. 85(6): p. 765-73.

15. Komaroff, A.L. and L. Bateman, Will COVID-19 Lead to Myalgic Encephalomyelitis/Chronic Fatigue Syndrome? Front Med (Lausanne), 2020. 7: p. 606824.

16. O'Sullivan, O., Long-term sequelae following previous coronavirus epidemics. Clin Med (Lond), 2021. 21(1): p. e68-e70.

17. Wostyn, P., COVID-19 and chronic fatigue syndrome: Is the worst yet to come? Med Hypotheses, 2021. 146: p. 110469.

18. Stefano, G.B., Historical Insight into Infections and Disorders Associated with Neurological and Psychiatric Sequelae Similar to Long COVID. Med Sci Monit, 2021. 27: p. e931447.

19. Writing Committee for the, C.S.G., et al., Four-Month Clinical Status of a Cohort of Patients After Hospitalization for COVID-19. JAMA, 2021. 
20. Sekirov, I., et al., Gut microbiota in health and disease. Physiol Rev, 2010. 90(3): p. 859904.

21. Galland, L., The gut microbiome and the brain. J Med Food, 2014. 17(12): p. 1261-72.

22. Zheng, D., T. Liwinski, and E. Elinav, Interaction between microbiota and immunity in health and disease. Cell Res, 2020. 30(6): p. 492-506.

23. Antonini, M., et al., How the Interplay Between the Commensal Microbiota, Gut Barrier Integrity, and Mucosal Immunity Regulates Brain Autoimmunity. Front Immunol, 2019. 10: p. 1937.

24. Du Preez, S., et al., A systematic review of enteric dysbiosis in chronic fatigue syndrome/myalgic encephalomyelitis. Syst Rev, 2018. 7(1): p. 241.

25. Nagy-Szakal, D., et al., Fecal metagenomic profiles in subgroups of patients with myalgic encephalomyelitis/chronic fatigue syndrome. Microbiome, 2017. 5(1): p. 44.

26. Fremont, M., et al., High-throughput 16S rRNA gene sequencing reveals alterations of intestinal microbiota in myalgic encephalomyelitis/chronic fatigue syndrome patients. Anaerobe, 2013. 22: p. 50-6.

27. Giloteaux, L., M.R. Hanson, and B.A. Keller, A Pair of Identical Twins Discordant for Myalgic Encephalomyelitis/Chronic Fatigue Syndrome Differ in Physiological Parameters and Gut Microbiome Composition. Am J Case Rep, 2016. 17: p. 720-729.

28. Giloteaux, L., et al., Reduced diversity and altered composition of the gut microbiome in individuals with myalgic encephalomyelitis/chronic fatigue syndrome. Microbiome, 2016. 4(1): p. 30 .

29. Kitami, T., et al., Deep phenotyping of myalgic encephalomyelitis/chronic fatigue syndrome in Japanese population. Sci Rep, 2020. 10(1): p. 19933. 
30. Lupo, G.F.D., et al., Potential role of microbiome in Chronic Fatigue Syndrome/Myalgic Encephalomyelits (CFS/ME). Sci Rep, 2021. 11(1): p. 7043.

31. Aaron, L.A., et al., Comorbid clinical conditions in chronic fatigue: a co-twin control study. J Gen Intern Med, 2001. 16(1): p. 24-31.

32. Hausteiner-Wiehle, C. and P. Henningsen, Irritable bowel syndrome: relations with functional, mental, and somatoform disorders. World J Gastroenterol, 2014. 20(20): p. 6024-30.

33. Kim, S.E. and L. Chang, Overlap between functional GI disorders and other functional syndromes: what are the underlying mechanisms? Neurogastroenterol Motil, 2012. 24(10): p. $895-913$.

34. Gupta, V.K., S. Paul, and C. Dutta, Geography, Ethnicity or Subsistence-Specific Variations in Human Microbiome Composition and Diversity. Front Microbiol, 2017. 8: p. 1162.

35. Chong, P.P., et al., The Microbiome and Irritable Bowel Syndrome - A Review on the Pathophysiology, Current Research and Future Therapy. Front Microbiol, 2019. 10: p. 1136.

36. Zhuang, X., et al., Fecal Microbiota Alterations Associated With Diarrhea-Predominant Irritable Bowel Syndrome. Front Microbiol, 2018. 9: p. 1600.

37. Rangel, I., et al., The relationship between faecal-associated and mucosal-associated microbiota in irritable bowel syndrome patients and healthy subjects. Aliment Pharmacol Ther, 2015. 42(10): p. 1211-21. 
38. Jeffery, I.B., et al., Differences in Fecal Microbiomes and Metabolomes of People With $v$ s Without Irritable Bowel Syndrome and Bile Acid Malabsorption. Gastroenterology, 2020. 158(4): p. 1016-1028 e8.

39. Ho, N.T., et al., metamicrobiomeR: an $R$ package for analysis of microbiome relative abundance data using zero-inflated beta GAMLSS and meta-analysis across studies using random effects models. BMC Bioinformatics, 2019. 20(1): p. 188.

40. Darzi, Y., et al., Towards biome-specific analysis of meta-omics data. ISME J, 2016. 10(5): p. $1025-8$.

41. Vital, M., A. Karch, and D.H. Pieper, Colonic Butyrate-Producing Communities in Humans: an Overview Using Omics Data. mSystems, 2017. 2(6).

42. Louis, P. and H.J. Flint, Development of a semiquantitative degenerate real-time pcr-based assay for estimation of numbers of butyryl-coenzyme A (CoA) CoA transferase genes in complex bacterial samples. Appl Environ Microbiol, 2007. 73(6): p. 2009-12.

43. Menees, S. and W. Chey, The gut microbiome and irritable bowel syndrome. F1000Res, 2018. 7.

44. Pimentel, M. and A. Lembo, Microbiome and Its Role in Irritable Bowel Syndrome. Dig Dis Sci, 2020. 65(3): p. 829-839.

45. Manichanh, C., et al., Reshaping the gut microbiome with bacterial transplantation and antibiotic intake. Genome Res, 2010. 20(10): p. 1411-9.

46. Panda, S., et al., Short-term effect of antibiotics on human gut microbiota. PLoS One, 2014. 9(4): p. e95476. 
47. Sloan, T.J., et al., A low FODMAP diet is associated with changes in the microbiota and reduction in breath hydrogen but not colonic volume in healthy subjects. PLoS One, 2018. 13(7): p. e0201410.

48. Kieser, S., et al., Antibiotic Treatment Leads to Fecal Escherichia coli and Coliphage Expansion in Severely Malnourished Diarrhea Patients. Cell Mol Gastroenterol Hepatol, 2018. 5(3): p. 458-460 e6.

49. Walujkar, S.A., et al., Characterization of bacterial community shift in human Ulcerative Colitis patients revealed by Illumina based $16 S$ rRNA gene amplicon sequencing. Gut Pathog, 2014. 6: p. 22.

50. Shah, A., et al., Duodenal bacterial load as determined by quantitative polymerase chain reaction in asymptomatic controls, functional gastrointestinal disorders and inflammatory bowel disease. Aliment Pharmacol Ther, 2020. 52(1): p. 155-167.

51. Louis, P., et al., Diversity of human colonic butyrate-producing bacteria revealed by analysis of the butyryl-CoA:acetate CoA-transferase gene. Environ Microbiol, 2010. 12(2): p. 304-14.

52. Duncan, S.H., et al., Acetate utilization and butyryl coenzyme A (CoA):acetate-CoA transferase in butyrate-producing bacteria from the human large intestine. Appl Environ Microbiol, 2002. 68(10): p. 5186-90.

53. Rios-Covian, D., et al., Enhanced butyrate formation by cross-feeding between Faecalibacterium prausnitzii and Bifidobacterium adolescentis. FEMS Microbiol Lett, 2015. 362(21).

54. Taras, D., et al., Reclassification of Eubacterium formicigenerans Holdeman and Moore 1974 as Dorea formicigenerans gen. nov., comb. nov., and description of Dorea 
longicatena sp. nov., isolated from human faeces. Int J Syst Evol Microbiol, 2002. 52(Pt 2): p. $423-428$.

55. Takada, T., et al., Fusicatenibacter saccharivorans gen. nov., sp. nov., isolated from human faeces. Int J Syst Evol Microbiol, 2013. 63(Pt 10): p. 3691-3696.

56. Sun, Q., et al., Alterations in fecal short-chain fatty acids in patients with irritable bowel syndrome: A systematic review and meta-analysis. Medicine (Baltimore), 2019. 98(7): p. e14513.

57. Mars, R.A.T., et al., Longitudinal Multi-omics Reveals Subset-Specific Mechanisms Underlying Irritable Bowel Syndrome. Cell, 2020. 183(4): p. 1137-1140.

58. LeBlanc, J.G., et al., Beneficial effects on host energy metabolism of short-chain fatty acids and vitamins produced by commensal and probiotic bacteria. Microb Cell Fact, 2017. 16(1): p. 79.

59. Parada Venegas, D., et al., Short Chain Fatty Acids (SCFAs)-Mediated Gut Epithelial and Immune Regulation and Its Relevance for Inflammatory Bowel Diseases. Front Immunol, 2019. 10: p. 277.

60. Schulthess, J., et al., The Short Chain Fatty Acid Butyrate Imprints an Antimicrobial Program in Macrophages. Immunity, 2019. 50(2): p. $432-445$ e7.

61. Miquel, S., et al., Faecalibacterium prausnitzii and human intestinal health. Curr Opin Microbiol, 2013. 16(3): p. 255-61.

62. Martin, R., L.G. Bermudez-Humaran, and P. Langella, Searching for the Bacterial Effector: The Example of the Multi-Skilled Commensal Bacterium Faecalibacterium prausnitzii. Front Microbiol, 2018. 9: p. 346. 
63. Leylabadlo, H.E., et al., The critical role of Faecalibacterium prausnitzii in human health: An overview. Microb Pathog, 2020. 149: p. 104344.

64. Quevrain, E., et al., Identification of an anti-inflammatory protein from Faecalibacterium prausnitzii, a commensal bacterium deficient in Crohn's disease. Gut, 2016. 65(3): p. 415425 .

65. Tang, L., et al., Clinical Significance of the Correlation between Changes in the Major Intestinal Bacteria Species and COVID-19 Severity. Engineering (Beijing), 2020. 6(10): p. 1178-1184.

66. Yeoh, Y.K., et al., Gut microbiota composition reflects disease severity and dysfunctional immune responses in patients with COVID-19. Gut, 2021. 70(4): p. 698-706.

67. Zhou, Y., et al., Linking the gut microbiota to persistent symptoms in survivors of COVID19 after discharge. J Microbiol, 2021.

68. Valles-Colomer, M., et al., The neuroactive potential of the human gut microbiota in quality of life and depression. Nat Microbiol, 2019. 4(4): p. 623-632.

69. Borren, N.Z., et al., Alterations in Fecal Microbiomes and Serum Metabolomes of Fatigued Patients With Quiescent Inflammatory Bowel Diseases. Clin Gastroenterol Hepatol, 2021. 19(3): p. 519-527 e5.

70. Vercoulen, J.H., et al., Physical activity in chronic fatigue syndrome: assessment and its role in fatigue. J Psychiatr Res, 1997. 31(6): p. 661-73.

71. van der Werf, S.P., et al., Identifying physical activity patterns in chronic fatigue syndrome using actigraphic assessment. J Psychosom Res, 2000. 49(5): p. 373-9.

72. Black, C.D., J. O'Connor P, and K.K. McCully, Increased daily physical activity and fatigue symptoms in chronic fatigue syndrome. Dyn Med, 2005. 4(1): p. 3. 
73. Kop, W.J., et al., Ambulatory monitoring of physical activity and symptoms in fibromyalgia and chronic fatigue syndrome. Arthritis Rheum, 2005. 52(1): p. 296-303.

74. Mailing, L.J., et al., Exercise and the Gut Microbiome: A Review of the Evidence, Potential Mechanisms, and Implications for Human Health. Exerc Sport Sci Rev, 2019. 47(2): p. $75-$ 85.

75. Clauss, M., et al., Interplay Between Exercise and Gut Microbiome in the Context of Human Health and Performance. Front Nutr, 2021. 8: p. 637010.

76. Smets, E.M., et al., The Multidimensional Fatigue Inventory (MFI) psychometric qualities of an instrument to assess fatigue. J Psychosom Res, 1995. 39(3): p. 315-25.

77. Martin, M., Cutadapt removes adapter sequences from high-throughput sequencing reads. EMBnet.journal, 2011. 17(1): p. 10-12.

78. Schmieder, R. and R. Edwards, Quality control and preprocessing of metagenomic datasets. Bioinformatics, 2011. 27(6): p. 863-4.

79. Langmead, B. and S.L. Salzberg, Fast gapped-read alignment with Bowtie 2. Nat Methods, 2012. 9(4): p. 357-9.

80. Lu, J. and S.L. Salzberg, Ultrafast and accurate $16 S$ rRNA microbial community analysis using Kraken 2. Microbiome, 2020. 8(1): p. 124.

81. Lu, J., et al., Bracken: estimating species abundance in metagenomics data. PeerJ Comput Sci, 2017. 3: p. 104.

82. Kaul, A., et al., Analysis of Microbiome Data in the Presence of Excess Zeros. Front Microbiol, 2017. 8: p. 2114.

83. Bolyen, E., et al., Reproducible, interactive, scalable and extensible microbiome data science using QIIME 2. Nat Biotechnol, 2019. 37(8): p. 852-857. 
84. Kim, J., et al., FMAP: Functional Mapping and Analysis Pipeline for metagenomics and metatranscriptomics studies. BMC Bioinformatics, 2016. 17(1): p. 420.

85. Ramirez-Farias, C., et al., Effect of inulin on the human gut microbiota: stimulation of Bifidobacterium adolescentis and Faecalibacterium prausnitzii. Br J Nutr, 2009. 101(4): p. 541-50.

86. Nadkarni, M.A., et al., Determination of bacterial load by real-time PCR using a broadrange (universal) probe and primers set. Microbiology (Reading), 2002. 148(Pt 1): p. 257266.

87. Moreau, N.M., et al., Simultaneous measurement of plasma concentrations and 13Cenrichment of short-chain fatty acids, lactic acid and ketone bodies by gas chromatography coupled to mass spectrometry. J Chromatogr B Analyt Technol Biomed Life Sci, 2003. 784(2): p. 395-403.

88. $\mathrm{Hu}, \mathrm{Y} . J$. and G.A. Satten, Testing hypotheses about the microbiome using the linear decomposition model (LDM). Bioinformatics, 2020. 36(14): p. 4106-4115.

89. Smirnova, E., S. Huzurbazar, and F. Jafari, PERFect: PERmutation Filtering test for microbiome data. Biostatistics, 2019. 20(4): p. 615-631. 


\section{Supplementary Files}

This is a list of supplementary files associated with this preprint. Click to download.

- MECFSSupplementalMaterials10252021.pdf 